TRANSACTIONS OF THE

AMERICAN MATHEMATICAL SOCIETY

Volume 352, Number 3, Pages 1405-1436

S 0002-9947(99)02258-8

Article electronically published on October 21, 1999

\title{
DOUBLE COSET DENSITY IN CLASSICAL ALGEBRAIC GROUPS
}

\author{
JONATHAN BRUNDAN
}

\begin{abstract}
We classify all pairs of reductive maximal connected subgroups of a classical algebraic group $G$ that have a dense double coset in $G$. Using this, we show that for an arbitrary pair $(H, K)$ of reductive subgroups of a reductive group $G$ satisfying a certain mild technical condition, there is a dense $H, K$ double coset in $G$ precisely when $G=H K$ is a factorization.
\end{abstract}

\section{INTRODUCTION}

In this paper, we consider the problem of classifying certain orbits of algebraic groups - double cosets. Let $H$ and $K$ be closed subgroups of a reductive algebraic group $G$, defined over an algebraically closed field of characteristic $p \geq 0$. Then, $H \times K$ acts on $G$ by $(x, y) \cdot g=x g y^{-1}$, for $(x, y) \in H \times K, g \in G$, and the orbits are the $H, K$-double cosets in $G$. We shall be concerned with the following properties:

(D1) $G=H K$ is a factorization of $G$.

(D2) There are finitely many $H, K$-double cosets in $G$.

(D3) There is a dense $H, K$-double coset in $G$.

Notice that $(\mathrm{D} 1) \Rightarrow(\mathrm{D} 2) \Rightarrow(\mathrm{D} 3)$. A primary aim motivating our work is to classify all triples $(G, H, K)$ satisfying (D2) or (D3). Factorizations - property (D1) - of simple algebraic groups, with $H$ and $K$ either reductive or parabolic, are classified in [LSS].

For example, take $H$ to be semisimple and $G=G L(V)$, where $V$ is some rational irreducible $H$-module. Let $K$ be the stabilizer in $G$ of a 1-subspace of $V$. Then, the $H, K$-double cosets in $G$ correspond naturally to the orbits of $H$ on 1-subspaces of $V$. This special case of the problem has been studied in some detail by a number of authors, both for $p=0$ and $p>0$. The irreducible modules on which $H$ has finitely many orbits have been classified [Kac, GLMS]. Similarly, the irreducible modules on which $H$ has a dense orbit have been classified [SK], [Ch1], [Ch2]. More generally, if $K$ is the stabilizer in $G$ of an $i$-dimensional subspace of $V$, the $H, K$ double cosets correspond to $H$-orbits on $i$-subspaces of $V$, also studied in [GLMS]; or if $H$ preserves a non-degenerate bilinear form on $V$, Guralnick and Seitz [GS] consider orbits of $H$ on degenerate $i$-subspaces of $V$, and this problem can also be reformulated easily as a problem about double cosets.

In all the cases just mentioned, $H$ is reductive and the subgroup $K$ in the double coset formulation of the problem is a parabolic subgroup of $G$. In this paper, we are concerned instead with the case that $H$ and $K$ are both reductive subgroups of $G$.

Received by the editors February 12, 1997 and, in revised form, September 17, 1997.

2000 Mathematics Subject Classification. Primary 20G15.

(C)1999 American Mathematical Society 
For example, this includes as a special case the study of orbits of a semisimple group $H$ on non-degenerate $i$-subspaces of a rational irreducible $H$-module possessing an $H$-invariant bilinear form. We now state the main results of this paper.

If $G$ is a connected reductive algebraic group, define $\mathcal{M}(G)$ to be the set of all maximal connected reductive subgroups of $G$ that are either Levi factors or maximal connected subgroups of $G$. Let $\mathcal{R}(G)$ be the set of reductive subgroups $H \leq G$ for which there is a chain of connected subgroups $H^{0}=H_{0}<H_{1}<\cdots<H_{n}=G$ such that for all $0 \leq i<n, H_{i} \in \mathcal{M}\left(H_{i+1}\right)$. So in particular, $\mathcal{R}(G)$ contains all reductive maximal subgroups of $G$ and all Levi factors, and if $p=0, \mathcal{R}(G)$ contains all reductive subgroups of $G$. Our main result is as follows:

Theorem A. Let $G$ be a connected reductive algebraic group, and take $H, K \in$ $\mathcal{R}(G)$. Then, either $G=H K$, or there is no dense $H, K$-double coset in $G$.

This shows that, under the hypothesis of Theorem A, properties (D1)-(D3) are equivalent. This contrasts with the case when we allow one of $H$ or $K$ to be parabolic, when there are examples (even if $p=0$ ) where each of the implications (D2) $\Rightarrow(\mathrm{D} 1)$ or - more unexpectedly - (D3) $\Rightarrow(\mathrm{D} 2)$ fails.

In $[\mathrm{Lu}]$, Luna shows that over algebraically closed fields of characteristic 0 , the union of the closed $H, K$-double cosets in $G$ is dense in $G$, for arbitrary reductive subgroups $H, K$ of a connected reductive group $G$. In particular, this implies that a dense $H, K$-double coset in $G$ must be closed, so that $G=H K$ is a factorization. Thus, Luna's stronger result implies Theorem A in characteristic 0 only. Luna's inductive proof depends on the construction of étale slices, which is always possible in characteristic zero thanks to complete reducibility of representations, but can often fail in small positive characteristic. It is possible to prove a partial version of Theorem A using Luna's methods - see [B1, chapter 1].

The approach here is quite different, based on knowledge of the maximal subgroups of simple algebraic groups. In the case that $G$ is simple of exceptional type, Theorem A follows from [B2]. The bulk of the work in this paper is in proving Theorem A in the case that $G$ is simple of classical type, which will follow from the next result:

Theorem B. Let $G$ be a simple classical algebraic group and $H, K \in \mathcal{M}(G)$. Then, either $G=H K$ is a factorization, or there is no dense $H, K$-double coset in $G$. In the former case, the triple $(G, H, K)$ is listed in Table 1.

The existence of every factorization in Table 1 follows from [LSS, Section 1]. Notation in the table will be explained in Section 1. The layout of the remainder of the paper is as follows. In section 1, we review some results on maximal subgroups of simple algebraic groups and the techniques developed in [B2]. The proof of Theorem B is given in sections 2, 3 and 4, and this is applied to prove Theorem A in section 5 .

\section{Preliminaries}

We set up notation and recall some well known general results in invariant theory. Then we review results of Seitz [Se] and Liebeck [L] concerning maximal subgroups of classical algebraic groups, which will be used in the proof of Theorem B.

1.1. Notation. Throughout, $k$ will be an algebraically closed field of characteristic $p \geq 0$, and $G$ will denote an affine algebraic group defined over $k$. Subgroups of 
TABLE 1. Maximal reductive factorizations of simple algebraic groups

\begin{tabular}{|l|l|l|l|}
\hline$G=C l(V)$ & $n, p$ & $H$ & $K$ \\
\hline \hline$S L_{2 n}$ & $n \geq 2$ & $S p_{2 n}$ & $L_{1}$ or $L_{2 n-1}$ \\
$S O_{2 n}$ & $n \geq 4$ & $N_{1}$ & $L_{n-1}$ or $L_{n}$ \\
$S O_{4 n}$ & $(n, p) \neq(2,2)$ & $N_{1}$ & $S p_{2} \otimes S p_{2 n}$ \\
$S p_{2 n}$ & $p=2$ & $N_{i}$ & $S O_{2 n}$ \\
\hline$S p_{6}$ & $p=2$ & $N_{2}, S O_{6}$ & $G_{2}, V \downarrow_{K}=L_{K}\left(\omega_{1}\right)$ \\
$S O_{7}$ & $p \neq 2$ & $L_{1}, N_{1}$ & $G_{2}, V \downarrow_{K}=L_{K}\left(\omega_{1}\right)$ \\
$S O_{8}$ & & $B_{3}$ & $L_{1}, L_{4}, N_{1}$ or $B_{3}$ \\
$S O_{8}$ & & $\tau_{3}$ & $L_{1}, L_{3}, N_{1}$ or $B_{3}$ \\
$S O_{8}$ & $p \neq 2$ & $B_{3}$ or ${ }^{\tau} B_{3}$ & $N_{3}$ or $S p_{2} \otimes S p_{4}$ \\
$S O_{13}$ & $p=3$ & $N_{1}$ & $C_{3}, V \downarrow_{K}=L_{K}\left(\omega_{2}\right)$ \\
$S O_{16}$ & & $N_{1}$ & $B_{4}, V \downarrow_{K}=L_{K}\left(\omega_{4}\right)$ \\
$S O_{20}$ & $p=2$ & $N_{1}$ & $A_{5}, V \downarrow_{K}=L_{K}\left(\omega_{3}\right)$ \\
$S O_{25}$ & $p=3$ & $N_{1}$ & $F_{4}, V \downarrow_{K}=L_{K}\left(\omega_{4}\right)$ \\
$S O_{32}$ & $p=2$ & $N_{1}$ & $D_{6}, V \downarrow_{K}=L_{K}\left(\omega_{5}\right)$ or $L_{K}\left(\omega_{6}\right)$ \\
$S O_{56}$ & $p=2$ & $N_{1}$ & $E_{7}, V \downarrow_{K}=L_{K}\left(\omega_{7}\right)$ \\
\hline
\end{tabular}

$G$ will always be assumed to be closed without further notice. By a $G$-module we mean a rational $k G$-module, and by a $G$-variety we mean an algebraic variety $X$ defined over $k$ on which $G$ acts morphically.

By a reductive algebraic group, we mean a (not necessarily connected) algebraic group $G$ with trivial unipotent radical. If $G$ is a connected reductive algebraic group, we define a root system of $G$ to be a quadruple $(T, B ; \Sigma, \Pi)$, where $T$ is a maximal torus of $G$ and $B$ is a Borel subgroup containing $T$. Let $X(T)=$ $\operatorname{Hom}\left(T, k^{\times}\right)$be the character group of $T$. The choice of $T$ determines a set of roots $\Sigma \subset X(T)$. For $\alpha \in \Sigma$, we shall write $U_{\alpha}$ for the corresponding $T$-root subgroup of $G$. The choice of $B$ determines a set of positive roots $\Sigma^{+}=\left\{\alpha \in \Sigma \mid U_{\alpha}<B\right\}$ and hence a base $\Pi$ for $\Sigma$.

Given a fixed root system $(T, B ; \Sigma, \Pi)$, we will adopt the following conventions.

Write $\Pi=\left\{\alpha_{1}, \ldots, \alpha_{n}\right\}$, where $n=\operatorname{rank} \Sigma$, and if $G$ is simple, label the simple roots $\alpha_{i}$ as in [H2]. Let $W=N_{G}(T) / T$ be the Weyl group of $G$, and choose a $W$-invariant inner product $\langle\cdot, \cdot\rangle$ on $\mathbb{R} \otimes_{\mathbb{Z}} X(T)$. Then $W$ is generated by $s_{1}, \ldots, s_{n}$, where $s_{i}$ is the simple reflection of $\mathbb{R} \otimes_{\mathbb{Z}} X(T)$ in the hyperplane orthogonal to $\alpha_{i}$. Fix fundamental dominant weights $\omega_{1}, \ldots, \omega_{n} \in \mathbb{R} \otimes_{\mathbb{Z}} X(T)$ such that

$$
\frac{2\left\langle\omega_{i}, \alpha_{j}\right\rangle}{\left\langle\alpha_{j}, \alpha_{j}\right\rangle}=\delta_{i j}
$$

Let $U$ be the unipotent radical of $B$, so $B=T U$. Let $B^{-}=T U^{-}$be the opposite Borel subgroup to $B$. For $w \in W$, let $U_{w}^{-}<U$ be the subgroup generated by root subgroups $U_{\alpha}$ such that $\alpha$ is a positive root sent to a negative root by $w$.

If $V$ is an arbitrary $G$-module and $\mu \in X(T)$, we shall write $V_{\mu}$ for the weight space $\{v \in V \mid t . v=\mu(t) v$ for all $t \in T\}$. When $G$ is semisimple and $\lambda$ is a dominant weight relative to some fixed root system of $G$, we shall write $L_{G}(\lambda)$ for the irreducible $G$-module of highest weight $\lambda$, and $\Delta_{G}(\lambda)$ for the corresponding Weyl module. Finally, we use $T_{i}$ (resp. $U_{i}$ ) to denote a torus (resp. a connected unipotent subgroup) of dimension $i$. 
For the first lemma, recall the properties (D1)-(D3) introduced in the introduction.

1.2. Lemma. Let $H$ and $K$ be subgroups of a connected algebraic group $G$. Let (D) be one of the properties (D1), (D2) or (D3).

(i) (D) holds for $(G, H, K)$ if and only if it holds for $\left(G, H^{0}, K^{0}\right)$. G.

(ii) (D) holds for $(G, H, K)$ if and only if it holds for $\left(G, H^{g}, K^{h}\right)$ for any $g, h \in$

(iii) Let $\theta: \tilde{G} \rightarrow G$ be a surjective morphism of algebraic groups, and set $\tilde{H}=\theta^{-1} H, \tilde{K}=\theta^{-1} K$. Then (D) holds for $(G, H, K)$ if and only if it holds for $(\tilde{G}, \tilde{H}, \tilde{K})$.

Proof. This is proved for (D1) in [LSS, Lemma 1.1]. The proofs for (D2), and of (i) and (ii) for (D3) are straightforward. So consider (iii) for (D3). Morphisms of algebraic groups are open maps, so any closed subset of $\tilde{G}$ which is a union of $\operatorname{ker} \theta$-cosets has closed image. Now, the closure of an $\tilde{H}, \tilde{K}$-double coset is a union of double cosets, hence a union of $\operatorname{ker} \theta$-cosets since $\operatorname{ker} \theta \leq \tilde{H}$. Hence, its image is also closed, and (iii) follows easily from this observation.

1.3. Some invariant theory. Let $G$ be an arbitrary algebraic group. If $X$ is a $G$-variety, the algebra of $G$-invariants on $X$ is defined to be

$$
k[X]^{G}:=\{f \in k[X] \mid g . f=f \text { for all } g \in G\},
$$

where $k[X]$ is the algebra of regular functions on $X$. Here, the action of $G$ on $k[X]$ is defined by $(g . f)(x):=f\left(g^{-1} x\right)$ for $g \in G, f \in k[X], x \in X$. If in addition $X$ is irreducible, we write $k(X)$ for the algebra of rational functions on $X$. The action of $G$ on $X$ also induces an action on $k(X)$, and we shall write $k(X)^{G}$ for the corresponding algebra of rational invariants.

In the case that $G$ is reductive and $X$ is an affine $G$-variety, the Mumford conjecture, proved in $[\mathrm{Hab}]$, plays a crucial role. For us, the most important consequence of the Mumford conjecture is the following lemma. We shall frequently use it to verify that there is no dense double coset in a given case in the proof of Theorem B.

1.4. Lemma ([B2, Lemma 2.1]). Suppose $G$ is reductive and $X$ is an affine $G$ variety. If $A$ and $B$ are disjoint closed $G$-stable subsets of $X$, then there exists an invariant $f \in k[X]^{G}$ with $f(a)=0$ for all $a \in A$ and $f(b)=1$ for all $b \in B$. In particular, if $G$ has at least two disjoint closed orbits in $X$, then there is no dense $G$-orbit in $X$.

To apply Lemma 1.4, we need to be able to prove that certain orbits are closed. Our main technique for this is the next elegant lemma, which is an easy consequence of the definition of a complete variety.

1.5. Lemma ([S2, p. 68 , Lemma 2]). Let $G$ act on a variety $X$, and let $P<G$ be a subgroup of $G$ such that $G / P$ is complete. If $U \subset X$ is closed and $P$-stable, then G.U is also closed.

Combining this with Lemma 1.4, it is easy to obtain the following results. See [B2, Section 2] for details.

1.6. Let $T$ be a maximal torus of $G$. Let $X$ be an affine $G$-variety, and suppose that $x \in X$ is fixed by T. Then, G.x is closed in $X$. 
1.7. Let $H$ and $K$ be reductive subgroups of $G$ with maximal tori $S, T$ respectively, such that $S \leq T$. Then, $H n K$ is closed in $G$ for all $n \in N_{G}(T)$.

1.8. Let $H$ be a proper reductive subgroup of a connected reductive algebraic group $G$. Then, there is no dense $H, H$-double coset in $G$.

Given a reductive group $G$ with root system $(T, B ; \Sigma, \Pi)$, there is a well-defined action of $W$ on the zero weight space $V_{0}$ of any $G$-module $V$. For later use, we record two useful lemmas:

1.9. Lemma ([B2, Lemma 4.1]). Let $V$ be a $G$-module and let $v, v^{\prime} \in V_{0}$. Then, $v$ and $v^{\prime}$ are conjugate under $G$ if and only if they are conjugate under $W$.

1.10. Lemma. Let $V$ be an irreducible $G$-module. Suppose that $G$ preserves a non-degenerate bilinear form on $V$. If $\mu, \nu$ are weights of $V$, then the weight spaces $V_{\mu}$ and $V_{\nu}$ are orthogonal unless $\mu=-\nu$. Hence, the restriction of the bilinear form to $V_{0}$ is non-degenerate.

Proof. Take $u \in V_{\mu}, v \in V_{\nu}$ such that $(u, v) \neq 0$. Then, for all $t \in T,(u, v)=$ $(t u, t v)=\mu(t) \nu(t)(u, v)$. Hence, $\mu(t) \nu(t)=1$, so $\mu=-\nu$ as required for the first part of the lemma. In particular, this shows that $V_{0}^{\perp}$ contains all non-zero weight spaces, and hence $V_{0}+V_{0}^{\perp}=V$. So, $V_{0}$ is indeed non-degenerate.

1.11. Maximal subgroups. Recall the notation $\mathcal{M}(G)$ from the introduction. We highlight at this point the difference between maximal connected reductive subgroups of $G$ and reductive maximal connected subgroups of $G$; the latter are maximal connected subgroups of $G$ that are also reductive, whereas the former may lie in some proper parabolic subgroup of $G$. If $G$ is connected and $p=0$, every maximal connected reductive subgroup of $G$ lies in $\mathcal{M}$, as a consequence of complete reducibility of representations. However, this need not be the case in arbitrary characteristic: there may be reductive subgroups of some parabolic subgroup $P$ of $G$ that lie in no Levi factor of $P$. This complication explains the need for the technical restriction that subgroups lie in $\mathcal{R}(G)$ or $\mathcal{M}(G)$ in Theorems A and B (though we believe that in fact no restriction is necessary).

We use the notation $G=C l(V)$ to indicate that $G$ is a connected classical algebraic group with natural module $V$. If $(G, p)=\left(B_{n}, 2\right)$, take $V$ to be the associated $2 n$-dimensional symplectic module. When $G=S O(V)$ or $S p(V)$, let $N_{i}$ denote the connected stabilizer in $G$ of a non-degenerate subspace of $V$ of dimension $i$ with $i \leq \frac{1}{2} \operatorname{dim} V$; and when $(G, p)=\left(D_{n}, 2\right)$, let $N_{1}$ denote the connected stabilizer of a non-singular 1-space.

1.12. Theorem ([Se, Theorem 3]). Let $G=C l(V)$, and suppose that $H$ is a reductive maximal connected subgroup of $G$. Then one of the following holds:

(i) $H=N_{i}$ for some $i$.

(ii) $V=U \otimes W$ and $H=C l(U) \otimes C l(W)$. 2).

(iii) $(G, H)=(S L(V), S p(V)),(S L(V), S O(V))(p \neq 2)$ or $(S p(V), S O(V))(p=$

(iv) $H$ is simple, and $V \downarrow_{H}$ is irreducible and tensor indecomposable, with $H \neq$ $C l(V)$.

When $G=C l(V)$, we let $\mathfrak{S}=\mathfrak{S}(G)$ be the set of all reductive maximal connected subgroups $H<G$ given by part (iv) of the theorem; so if $H \in \mathfrak{S}$, then $H$ is a simple maximal connected subgroup of $G=C l(V)$ and $V \downarrow_{H}$ is irreducible and tensor indecomposable. 
TABLE 2. Modules of small dimension

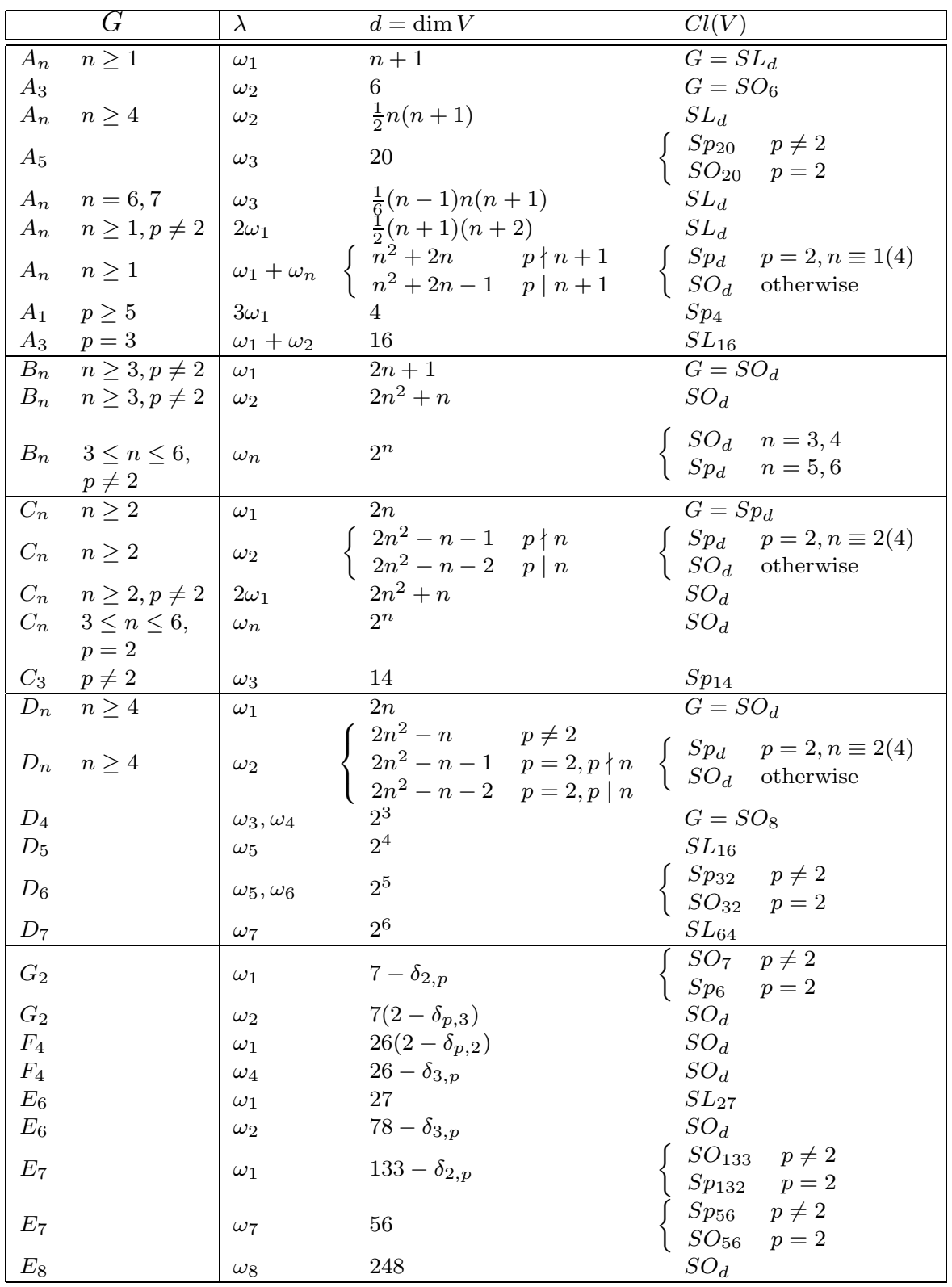

We introduce the notation $P_{i}$ to denote the maximal parabolic subgroup obtained by deleting the $i$ th node from the Dynkin diagram of $G$, and $L_{i}$ to denote a Levi factor of $P_{i}$. Explicitly, if $(T, B ; \Sigma, \Pi)$ is a root system for $G$, then we may take $P_{i}=\left\langle B, U_{-\alpha_{j}} \mid j \neq i\right\rangle$ and $L_{i}=\left\langle T, U_{ \pm \alpha_{j}} \mid j \neq i\right\rangle$.

We have now explained most of the notation in Table 1 . There are some finer points still to be explained. In $G=S O_{8}$, there are three classes of subgroup of type $B_{3}$. We denote representatives of these classes by $B_{3},{ }^{\tau} B_{3}$ and $N_{1}$ in Table 1 . Here, $B_{3}$ denotes a subgroup of $G$ of type $B_{3}$ such that $L_{G}\left(\omega_{1}\right) \downarrow_{B_{3}}=L_{G}\left(\omega_{3}\right) \downarrow_{B_{3}}=$ 
$L_{B_{3}}\left(\omega_{3}\right)$, whilst ${ }^{\tau} B_{3}$ denotes a subgroup of $G$ of type $B_{3}$ such that $L_{G}\left(\omega_{1}\right) \downarrow_{\tau_{B_{3}}}=$ $L_{G}\left(\omega_{4}\right) \downarrow_{\tau_{B_{3}}}=L_{B_{3}}\left(\omega_{3}\right)$. If in $P S O_{8}$, we let $\tau$ be a triality automorphism that induces the permutation $\left(\omega_{1} \omega_{3} \omega_{4}\right)$ on the fundamental weights $\left\{\omega_{1}, \ldots, \omega_{4}\right\}$, then $\tau$ induces the permutation $\left(B_{3}{ }^{\tau} B_{3} N_{1}\right)$ on the images of $B_{3},{ }^{\tau} B_{3}$ and $N_{1}$ in $P S O_{8}$. Later, we shall refer to $B_{3}<S O_{8}=G$ such that $L_{G}\left(\omega_{1}\right) \downarrow_{B_{3}}=L_{B_{3}}\left(\omega_{3}\right)$. This is ambiguous and should be taken to mean either $B_{3}$ or ${ }^{\tau} B_{3}$. In a similar fashion, we shall refer to $G L_{n}<S O_{2 n}$. By this we mean either of the two classes $L_{n-1}$ or $L_{n}$ of subgroups of $S O_{2 n}$ of type $G L_{n}$. Likewise $S p_{2} \otimes S p_{2 n}<S O_{4 n}$.

1.13. Modules of small dimension. To list the subgroups in $\mathfrak{S}(G)$ for $G$ classical, of large dimension relative to $\operatorname{dim} G$, we require some known information on modules for simple algebraic groups of small dimension relative to the dimension of the group. Let $G$ denote a simple algebraic group with fixed root system $(T, B ; \Sigma, \Pi)$. If $p=2$, we assume in addition that $G$ is not of type $B_{n}$; we may make this assumption without loss of generality because of the existence of bijective morphisms (which are not isomorphisms of algebraic groups) $B_{n} \rightarrow C_{n}$ and $C_{n} \rightarrow B_{n}$ in characteristic 2. We define numbers $e_{G}$ to be

\begin{tabular}{l|lllllllll}
$G$ & $A_{n}$ & $B_{n}$ & $C_{n}$ & $D_{n}$ & $G_{2}$ & $F_{4}$ & $E_{6}$ & $E_{7}$ & $E_{8}$ \\
\hline$e_{G}$ & $m+3$ & $m+n+1$ & $m+2$ & $m+n$ & 18 & 96 & 80 & 192 & 1024
\end{tabular}

where $m=\operatorname{dim} G$.

If $V$ is an irreducible $G$-module, we define $C l(V)$ to be the smallest classical group on $V$ containing the image of $G$ in $G L(V)$ (this is well-defined as $V$ is irreducible).

1.14. Theorem. Let $V=L_{G}(\lambda)$ be an irreducible, tensor indecomposable $G$ module such that $1<\operatorname{dim} V<e_{G}$. Then, up to duals and field twists, the quadruple $(G, \lambda, \operatorname{dim} V, C l(V))$ is listed in Table 2.

We remark that some care is needed interpreting Table 2 if $G=C_{n}$ and $p=2$. In this case, the image of $G$ in $G L(V)$ according to the representations in Table 2 need not be of type $C_{n}$. For example, the image of $C_{n}$ in a spin representation in characteristic 2 is isomorphic to $B_{n}$ as an algebraic group.

Apart from the information on $C l(V)$, this theorem follows immediately from [L, Section 2]. To compute $C l(V)$, the methods of [LSS, Section 2] suffice unless $p=2$ and $V$ is a self-dual composition factor of $\operatorname{Lie}(G)$. The result for this final possibility follows from results of Gow and Willems [GW] (or see [B1, chapter 2]).

We now give a first application of the information in Table 2.

1.15. Lemma. Let $G=C l(V)$ a classical algebraic group and $H<G$ be a reductive maximal connected subgroup with $\operatorname{dim} H \geq \frac{1}{2} \operatorname{dim} G$. Then $(G, H)$ are listed below:

\begin{tabular}{l|l|l}
$G$ & $H$ & Conditions \\
\hline$C l(V)$ & $N_{i}$ & \\
$S L(V)$ & $S p(V)$ & \\
$S p(V)$ & $S O(V)$ & $p=2$ \\
$S O_{8}$ & $B_{3}$ & $V \downarrow_{H}=L_{H}\left(\omega_{3}\right)$ \\
$S O_{7}(p \neq 2), S p_{6}(p=2)$ & $G_{2}$ & $V \downarrow_{H}=L_{H}\left(\omega_{1}\right)$
\end{tabular}


Proof. We apply Lemma 1.12, to see that either $(G, H)$ is as in the conclusion or $H \in \mathfrak{S}(G)$. In the latter case, note that $\operatorname{dim} H \geq \frac{1}{2} \operatorname{dim} G \geq \operatorname{dim} V$, so the pair $\left(H, V \downarrow_{H}\right)$ is listed in Table 2. Also, $G$ is the group $C l(V)$ listed in the table, since $H$ is maximal in $G$. Thus, checking dimensions for each possibility in Table 2 gives the conclusion.

\section{Proof of Theorem B: Maximal Reductive subgroups}

We are now ready to prove Theorem B. The strategy is as follows. We first use the information on maximal subgroups and modules of small dimension in Section 1 to list all pairs $(H, K)$ of subgroups in $\mathcal{M}(G)$ satisfying the dimension bound $\operatorname{dim} H+\operatorname{dim} K \geq \operatorname{dim} G$. We then verify each case in turn, using Lemma 1.4. We divide the case analysis into two halves. In this and the next section, we consider the possibilities when both $H$ and $K$ are maximal reductive connected subgroups, and in section 4 we consider the remaining possibilities when one of $H$ or $K$ is a Levi factor.

2.1. Proposition. Let $G$ be simple and $H, K \in \mathcal{M}(G)$ with both $H, K$ reductive maximal connected subgroups. To prove Theorem $B$ for the triple $(G, H, K)$, it is sufficient to show that it holds for $(G, H, K)$ in Table 3. (In the table we reference the lemma in which we treat these subgroups.)

Proof. Let $G=C l(V)$, and let $H, K$ be reductive maximal connected subgroups of $G$. If $H, K$ are conjugate, then there is no dense $H, K$-double coset in $G$ by (1.8), and if $\operatorname{dim} H+\operatorname{dim} K<\operatorname{dim} G$ then there can be no dense double coset by dimension. Thus, we may assume $H, K$ are not conjugate and that $\operatorname{dim} H+\operatorname{dim} K \geq$ $\operatorname{dim} G$. Moreover, if $(G, p)=\left(B_{n}, 2\right)$, then we can apply a bijective morphism $B_{n} \rightarrow C_{n}$ to deduce the result for $B_{n}$ from the corresponding result for $C_{n}$. Hence, we will assume $(G, p) \neq\left(B_{n}, 2\right)$. We now apply Theorem 1.12 to list all possibilities meeting these conditions. This is easy, but rather lengthy, so we only sketch the argument.

We may assume $\operatorname{dim} H \geq \operatorname{dim} K$, so $\operatorname{dim} H \geq \frac{1}{2} \operatorname{dim} G$. Hence, $(G, H)$ is given by Lemma 1.15; we consider the possibilities one by one.

(a) $(G, H)=\left(C l(V), N_{i}\right)$. List the possibilities for $K$, using Theorem 1.12 to see that one of the following holds: (i) $(G, K)=\left(C l(V), N_{j}\right)$; (ii) $(G, K)=$ $(C l(V), C l(U) \otimes C l(W))$ where $V=U \otimes W$; (iii) $(G, K)=(S p(V), S O(V))(p=2)$; (iv) $K \in \mathfrak{S}(G)$. Cases (i)-(iii) are all listed in Table 3. So, consider case (iv). First, suppose $i=1$, so $(G, H)=\left(S O(V), N_{1}\right)$ and $K \in \mathfrak{S}(G)$ with $\operatorname{dim} K \geq \operatorname{dim} G-$ $\operatorname{dim} H=\operatorname{dim} V-1$. So, $\left(K, V \downarrow_{K}, \operatorname{dim} V, G\right)$ is in Table 2. Hence, either $V \downarrow_{K}$ is a composition factor of $\operatorname{Lie}(K)$, or $(K, \lambda)=\left(A_{5}, \omega_{3}\right)(p=2),\left(B_{n}, \omega_{n}\right)(n=3,4)$, $\left(B_{n}, \omega_{n}\right)(p=2, n=5,6),\left(D_{6}, \omega_{6}\right)(p=2),\left(G_{2}, \omega_{1}\right)(p \neq 2),\left(F_{4}, \omega_{4}\right)(p \neq 2)$ or $\left(E_{7}, \omega_{7}\right)$. All of these are included in Table 3 except for $B_{5}$ in $p=2$, which lies in $D_{6}$ so is not maximal.

Now suppose $i \geq 2$. Note that if $G=S O(V)$, then $i \neq 2$ as $N_{2}$ is not maximal in $S O(V)$. Hence, $\operatorname{dim} K \geq \operatorname{dim} G-\operatorname{dim} H$ implies either $\operatorname{dim} K \geq$ $2 \operatorname{dim} V-4$ if $G=S p(V)$, or $\operatorname{dim} K \geq 3 \operatorname{dim} V-9$ if $G=S O(V)$. In particular, $\left(K, V \downarrow_{K}, \operatorname{dim} V, G\right)$ is in Table 2. Listing the possibilities that meet the dimension bound on $\operatorname{dim} K$, we deduce $(K, \lambda)=\left(G_{2}, \omega_{1}\right)$ (or $\left(G_{2}, \omega_{2}\right)$ if $p=3$, which yields the same embedding), $\left(B_{3}, \omega_{3}\right),\left(D_{6}, \omega_{6}\right)(p \neq 2)$, or $\left(E_{7}, \omega_{7}\right)(p \neq 2)$. Now for each 
TABLE 3. Case list involving maximal subgroups

\begin{tabular}{|l|l|l|l|c|}
\hline$G$ & $p$ & $H$ & $K$ & Ref \\
\hline \hline$C l(V)$ & & $N_{i}$ & $N_{j}$ & 2.2 \\
$C l(V)$ & & $N_{i}$ & $C l(U) \otimes C l(W), V=U \otimes W$ & 3.1 \\
$S L(V)$ & $p \neq 2$ & $S p(V)$ & $S O(V)$ & 2.4 \\
$S p(V)$ & $p=2$ & $N_{i}$ & $S O(V)$ & {$[\mathrm{LSS}]$} \\
$S O(V)$ & & $N_{1}$ & $K \in \mathfrak{S}(G), V \downarrow_{K}$ a composition & 2.5 \\
& & & factor of Lie $(K)$ & \\
$S O(V)$ & $p \neq 2$ & $N_{1}$ & $C_{n}, V \downarrow_{K}=L_{K}\left(\omega_{2}\right)$ & 2.5 \\
$S p(V)$ & $p=2$ & $S O(V)$ & $C_{n}, V \downarrow_{K}=L_{K}\left(\omega_{2}\right)$ and $n \equiv 2(4)$ & 2.6 \\
$S p(V)$ & $p=2$ & $S O(V)$ & $A_{n}, V \downarrow_{K}=L_{K}\left(\omega_{1}+\omega_{n}\right)$ and $n \equiv 1(4)$ & 2.6 \\
\hline$S p_{6}$ & $p=2$ & $S O_{6}$ or $N_{2}$ & $G_{2}, V \downarrow_{K}=L_{K}\left(\omega_{1}\right)$ & {$[\mathrm{LSS}]$} \\
$S O_{7}$ & $p \neq 2$ & $N_{1}$ or $N_{3}$ & $G_{2}, V \downarrow_{K}=L_{K}\left(\omega_{1}\right)$ & 2.3 \\
$S O_{8}$ & & $N_{1}$ or $N_{3}$ & $B_{3}, V \downarrow_{K}=L_{K}\left(\omega_{3}\right)$ & {$[\mathrm{LSS}]$} \\
$S O_{16}$ & & $N_{1}$ & $B_{4}, V \downarrow_{K}=L_{K}\left(\omega_{4}\right)$ & {$[\mathrm{LSS}]$} \\
$S O_{20}$ & $p=2$ & $N_{1}$ & $A_{5}, V \downarrow_{K}=L_{K}\left(\omega_{3}\right)$ & {$[\mathrm{LSS}]$} \\
$S O_{26-\delta_{p, 3}}$ & & $N_{1}$ & $F_{4}, V \downarrow_{K}=L_{K}\left(\omega_{4}\right)$ & 2.5 \\
$S p_{32}$ & $p \neq 2$ & $N_{2}$ & $D_{6}, V \downarrow_{K}=L_{K}\left(\omega_{6}\right)$ & {$[\mathrm{LSS}]$} \\
$S O_{32}$ & $p=2$ & $N_{1}$ & $D_{6}, V \downarrow_{K}=L_{K}\left(\omega_{6}\right)$ & 2.7 \\
$S p_{56}$ & $p \neq 2$ & $N_{2}$ & $E_{7}, V \downarrow_{K}=L_{K}\left(\omega_{7}\right)$ & {$[\mathrm{LSS}]$} \\
$S O_{56}$ & $p=2$ & $N_{1}$ & $E_{7}, V \downarrow_{K}=L_{K}\left(\omega_{7}\right)$ & 2.8 \\
$S O_{64}$ & $p=2$ & $N_{1}$ & $B_{6}, V \downarrow_{K}=L_{K}\left(\omega_{6}\right)$ \\
$S p_{132}$ & $p=2$ & $S O_{132}$ & $E_{7}, V \downarrow_{K}=L_{K}\left(\omega_{1}\right)$ & 2.6 \\
\hline
\end{tabular}

of these cases, one computes the permissible values of $i$, to obtain the entries in Table 3: $(G, H, K)=\left(S O_{7}, N_{3}, G_{2}\right) \quad(p \neq 2),\left(S p_{6}, N_{2}, G_{2}\right) \quad(p=2),\left(S O_{8}, N_{3}, B_{3}\right)$, $\left(S p_{32}, N_{2}, D_{6}\right) \quad(p \neq 2)$ and $\left(S p_{56}, N_{2}, E_{7}\right) \quad(p \neq 2)$.

(b) $(G, H)=(S L(V), S p(V))$. List the possibilities for $K$ to obtain: (i) $K=$ $S O(V)$; (ii) $K=C l(U) \otimes C l(W)$ where $V=U \otimes W$; (iii) $K \in \mathfrak{S}(G)$. Case (i) is listed in the table, and case (ii) does satisfy the dimension bound $\operatorname{dim} H+\operatorname{dim} K \geq \operatorname{dim} G$. In case (iii), $\operatorname{dim} K \geq \operatorname{dim} G-\operatorname{dim} H$ implies $\operatorname{dim} K \geq \frac{1}{2} d(d-1)-1$ where $d=\operatorname{dim} V$, which is even. So, $\left(K, V \downarrow_{K}, d, G\right)$ is as in Table 2. Considering the cases in the table one by one, none satisfy the requirements.

(c) $(G, H)=(S p(V), S O(V))(p=2)$. By (a), we may assume $K \neq N_{i}$. Listing the remaining possibilities, we see: (i) $K=C l(U) \otimes C l(W)$ where $V=U \otimes W$; (ii) $K \in \mathfrak{S}(G)$. Case (i) does not occur, as $C l(U) \otimes C l(W)$ preserves a quadratic form on $V$ if $p=2$, so is not maximal. In case (ii), $\operatorname{dim} K \geq \operatorname{dim} G-\operatorname{dim} H$ implies $\operatorname{dim} K \geq \operatorname{dim} V$, so $\left(K, V \downarrow_{K}, \operatorname{dim} V, G\right)$ is in Table 2. Listing the possibilities, we see that $V \downarrow_{K}=L_{K}(\lambda)$, where $(K, \lambda)=\left(A_{n}, \omega_{1}+\omega_{n}\right)(n \equiv 1(4)),\left(C_{n} / D_{n}, \omega_{2}\right)(n \equiv$ $2(4)),\left(G_{2}, \omega_{1}\right)$ or $\left(E_{7}, \omega_{1}\right)$. All of these are included in Table 3 except for $D_{n}$, which is not maximal.

(d) $(G, H)=\left(S O_{8}, B_{3}\right)$, where $V \downarrow_{H}=L_{B_{3}}\left(\omega_{3}\right),\left(S O_{7}, G_{2}\right)(p \neq 2)$ or $\left(S p_{6}, G_{2}\right)$ $(p=2)$. If $H=B_{3}$, we can deduce the result from the case $H=N_{1}$ by applying a triality automorphism to send $B_{3} \rightarrow N_{1}$; one needs to work in $\mathrm{PSO}_{8}$ here since triality is not defined in $S_{8}$ if $p \neq 2$. Otherwise, $H=G_{2}$ and $K \in \mathfrak{S}(G)$, and listing the possibilities for $K$ using Table 2, one concludes $K$ is conjugate to a subgroup of $H$ in all cases. 
Recall the definition of transporter: if $V$ is a $G$-variety and $A, B \subset V$ are subsets with $B$ closed, then $\operatorname{Tran}_{G}(A, B)=\{g \in G \mid g . A \subset B\}$ is a closed subset of $G$.

We now prove Theorem B for all cases in Table 2 except $\left(C l(V), N_{i}, C l(U) \otimes\right.$ $C l(W))$, which we postpone to Section 3 .

2.2. Lemma. There is no dense $H, K$-double coset in $G$ if

$$
(G, H, K)=\left(C l(V), N_{i}, N_{j}\right) .
$$

Proof. We may assume $j \neq i$, since otherwise $H$ and $K$ are conjugate and the result holds by (1.8). So, let $\operatorname{dim} V=n$ and $1 \leq j<i \leq \frac{1}{2} n$. Then, $G=S p(V)$ or $S O(V), H$ is the stabilizer of a non-degenerate subspace $V_{H}$ of dimension $i$, and $K$ is the stabilizer of a non-degenerate subspace $V_{K}$ of dimension $j$ (or if $G=S O(V)$, $p=2$ and $j=1, K$ is the stabilizer of a non-singular line $V_{K}$ ). Conjugating, we may assume that $V_{K}<V_{H}$.

We claim that $H K=\operatorname{Tran}_{G}\left(V_{K}, V_{H}\right)$. One inclusion is obvious, so take $g$ to be an element of $\operatorname{Tran}_{G}\left(V_{K}, V_{H}\right)$. Then, $g V_{K}$ is a non-degenerate (or non-singular) subspace of $V_{H}$ of dimension $j ; H$ acts transitively on these, so we can find $x \in H$ such that $x g V_{K}=V_{K}$. Hence, $x g \in K$ and $g \in H K$. Thus, $H K=\operatorname{Tran}_{G}\left(V_{K}, V_{H}\right)$, and in particular, $H K$ is closed.

Now, pick $h \in G$ such that $h V_{K}<V_{H}^{\perp}$. Then, $h K h^{-1}$ is the stabilizer of the subspace $h V_{K}$, and, by an identical argument, $H h K h^{-1}=\operatorname{Tran}_{G}\left(h V_{K}, V_{H}^{\perp}\right)$, so $H h K=\operatorname{Tran}_{G}\left(V_{K}, V_{H}^{\perp}\right)$, which is closed. Also, $\operatorname{Tran}_{G}\left(V_{K}, V_{H}\right)$ and $\operatorname{Tran}_{G}\left(V_{K}, V_{H}^{\perp}\right)$ are clearly disjoint. So, $H K$ and $H h K$ are disjoint closed $H, K$-double cosets in $G$, and the result follows by Lemma 1.4.

\subsection{Lemma. Theorem $B$ holds if}

$$
\begin{aligned}
& (G, H, K)=\left(S O_{7}, N_{1}, G_{2}\right), \\
& (G, H, K)=\left(S O_{7}, N_{3}, G_{2}\right),
\end{aligned}
$$

where $V \downarrow_{G_{2}}=L_{G_{2}}\left(\omega_{1}\right)$ and $p \neq 2$.

Proof. The case $(G, H, K)=\left(S_{7}, N_{1}, G_{2}\right)$ is a factorization by [LSS]. So, we just need to consider $(G, H, K)=\left(S_{7}, N_{3}, G_{2}\right)$.

Let $V$ be the spin module for $G=B_{3}$, so $V=L_{B_{3}}\left(\omega_{3}\right)$. Fixing a maximal torus $T$ of $G$, we will write a base for $G$ relative to $T$ as $\left\{\varepsilon_{1}-\varepsilon_{2}, \varepsilon_{2}-\varepsilon_{3}, \varepsilon_{3}\right\}$ in the usual way. Then, the non-zero weight spaces in $V$ correspond to weights $\left\{\frac{1}{2}\left( \pm \varepsilon_{1} \pm \varepsilon_{2} \pm \varepsilon_{3}\right)\right\}$; we abbreviate $\frac{1}{2}\left(+\varepsilon_{1}+\varepsilon_{2}+\varepsilon_{3}\right)$ as +++ , etc. Let $H=N_{3}$, a maximal rank subgroup of $G$ which may be chosen to be generated by root subgroups $U_{ \pm\left(\varepsilon_{1}-\varepsilon_{2}\right)}, U_{ \pm\left(\varepsilon_{1}+\varepsilon_{2}\right)}$ and $U_{ \pm \varepsilon_{3}}$. Let $V_{H}$ be the span of weight spaces corresponding to weights,,,+++++------+ , which is $H$-stable since each of the root group generators of $H$ stabilizes $V_{H}$. The restriction of the form on $V$ to $V_{H}$ is non-degenerate by Lemma 1.10 , so $V=V_{H} \oplus V_{H}^{\perp}$ is a direct sum of $H$-modules. Let $V_{K}$ be a non-degenerate line in $V_{H}$, and set $K=\operatorname{stab}_{G}\left(V_{K}\right)$, a subgroup of type $G_{2}$ as $G_{2}=B_{3} \cap N_{1}$ in $S O(V)$.

We know $S O(V)=B_{3} N_{1}$, so $G$ acts transitively on non-degenerate lines in $V$

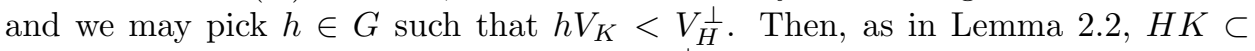
$\operatorname{Tran}_{G}\left(V_{K}, V_{H}\right)$ whilst $H h K \subset \operatorname{Tran}_{G}\left(V_{K}, V_{H}^{\perp}\right)$. These transporters are closed and disjoint, so contain disjoint closed $H, K$-double cosets to complete the proof by Lemma 1.4. 
2.4. Lemma. There is no dense $H, K$-double coset in $G$ if

$$
(G, H, K)=(S L(V), S p(V), S O(V))(p \neq 2) .
$$

Proof. The proof of this case is identical to the proof for $(G, H, K)=\left(E_{6}, F_{4}, C_{4}\right)$ in [B2, Lemma 6.1].

2.5. Lemma. Theorem $B$ holds if $G=S O(V), H=N_{1}$ and $K \in \mathfrak{S}$, where $V \downarrow_{K}=$ $L_{K}(\lambda)$ is either a composition factor of the adjoint module of $K$ or $(K, \lambda)=\left(F_{4}, \omega_{4}\right)$ or $\left(C_{n}, \omega_{2}\right)$.

Proof. Notice here we are excluding $K=A_{n}(n \equiv 1(4)), C_{n}(n \equiv 2(4))$ and $E_{7}$ when $p=2$, as then $K$ is not a subgroup of $G$ by [GW]. Let $T$ be a maximal torus of $K$. If $(G, H, K, p)=\left(S O_{25}, N_{1}, F_{4}, 3\right)$ or $\left(S O_{13}, N_{1}, C_{3}, 3\right)$, then $G=H K$ is a factorization by [LSS]. Otherwise, $\operatorname{dim} V_{0} \geq 2$. Hence, since by Lemma 1.10, the restriction of the bilinear form to $V_{0}$ is non-degenerate, there are infinitely many vectors of length 1 in $V_{0}$. By Lemma 1.9, there must therefore be infinitely many non-conjugate vectors of length 1 in $V_{0}$. Furthermore, by (1.6), these all have closed $K$-orbits. Thus, under the usual identification between $H, K$-double cosets in $G$ and $K$-orbits on vectors of length 1 in $V$, we have found infinitely many closed $H, K$-double cosets, and the result follows by Lemma 1.4 again.

2.6. Lemma. There is no dense $H, K$-double coset in $G$ if $p=2$ and

$$
\begin{aligned}
& (G, H, K, \lambda)=\left(S p(V), S O(V), A_{n}, \omega_{1}+\omega_{n}\right)(n \equiv 1(4)), \\
& (G, H, K, \lambda)=\left(S p(V), S O(V), C_{n}, \omega_{2}\right)(n \equiv 2(4)), \\
& (G, H, K, \lambda)=\left(S p(V), S O(V), E_{7}, \omega_{1}\right),
\end{aligned}
$$

where $V \downarrow_{K}=L_{K}(\lambda)$.

Proof. Let $d=\operatorname{dim} V$. Let $W$ be a $(d+2)$-dimensional orthogonal space with quadratic form $Q$, and fix a non-singular line $\langle w\rangle$ where $Q(w)=1$. Let $\bar{G} \cong$ $S O_{d+1}$ be the stabilizer of $\langle w\rangle$ in $S O(W)$. Then, $V=\langle w\rangle^{\perp} /\langle w\rangle$ is a $d$-dimensional symplectic space, and the map $\langle w\rangle^{\perp} \rightarrow V$ induces a bijective morphism $\bar{G} \rightarrow G \cong$ $S p_{d}$. Let $\bar{H}, \bar{K}$ be the pre-images of $H, K$ respectively. It will be sufficient to show that there are disjoint closed $\bar{H}, \bar{K}$-double cosets in $\bar{G}$, because of Lemma 1.2(iii). As $\bar{H} \cong S O_{d}$, it stabilizes some complement $U$ to $\langle w\rangle$ in $\langle w\rangle^{\perp}$. Let $Z=\left(\langle w\rangle^{\perp}\right)^{*}$ and note that $\bar{H}$ is the stabilizer in $\bar{G}$ of the vector $f_{0} \in Z$, where $f_{0}(w)=1, f_{0}(U)=0$. Hence, it is sufficient to show that $\bar{K}$ has disjoint closed orbits in $\bar{G} . f_{0}$.

We claim that $\bar{G} \cdot f_{0}=\{f \in Z \mid f(w)=1\}$. To prove this, it is sufficient to show that $\bar{G}$ acts transitively on complements to $\langle w\rangle$ in $\langle w\rangle^{\perp}$, or equivalently that $\bar{G}$ acts transitively on non-degenerate 2 -spaces in $W$ containing $\langle w\rangle$. This follows easily by Witt's lemma.

Let $T$ be a maximal torus of $\bar{H}$. Then, $\operatorname{dim} Z_{0}=\operatorname{dim}\left(\langle w\rangle^{\perp}\right)_{0}=\operatorname{dim} V_{0}+1$. Hence, since $K \neq A_{1}$ or $C_{2}$ (when $K=G$ ), $\operatorname{dim} Z_{0} \geq 2$. So there are infinitely many vectors of weight 0 in $\bar{G}$. $f_{0}$, and the result follows by (1.6) and Lemma 1.9.

The proof of the next lemma is based on the proof that $A_{1} D_{6}$ (resp. $A_{1} E_{7}$ ) has infinitely many orbits on $L_{A_{1}}\left(\omega_{1}\right) \otimes L_{D_{6}}\left(\omega_{6}\right)$ (resp. $L_{A_{1}}\left(\omega_{1}\right) \otimes L_{E_{7}}\left(\omega_{7}\right)$ ) in [GLMS]. We also treat the case $N_{2}$ in $S O(V)$ here for later use, even though it is not maximal. 
2.7. Lemma. There is no dense $H, K$-double coset in $G$ if

$$
\begin{aligned}
& (G, H, K)=\left(S p(V), N_{2}, D_{6}\right) \text { or }\left(S p(V), N_{2}, E_{7}\right) \text { and } p \neq 2, \\
& (G, H, K)=\left(S O(V), N_{2}, D_{6}\right) \text { or }\left(S O(V), N_{2}, E_{7}\right) \text { and } p=2,
\end{aligned}
$$

where $V \downarrow_{K}=L_{K}\left(\omega_{6}\right)$ if $K=D_{6}$ or $L_{K}\left(\omega_{7}\right)$ if $K=E_{7}$.

Proof. Let $d=\operatorname{dim} V$. Let $U$ be a 2-dimensional symplectic space, and set $W=U \otimes$ $V$, a $2 d$-dimensional orthogonal space. Write $(\cdot, \cdot)$ for the bilinear forms on $U, V, W$. Let $L=S p(U) \otimes K \cong A_{1} D_{6}$ or $A_{1} E_{7}$, a subgroup of $S O(W)$. Let $A$ be the $A_{1}$ factor of $L$. Let $\Omega_{1}=\{w \in W \mid(w, w)=1\}$ and $\Omega_{2}=\left\{v_{1} \wedge v_{2} \in \wedge^{2} V \mid\left(v_{1}, v_{2}\right)=1\right\}$. To prove the lemma, it will be sufficient to show that $K$ has at least two disjoint closed orbits in $\Omega_{2}$.

Let $e, f$ be a basis for $U$ with $(e, f)=1$. Any vector $w \in W$ with $(w, w)=1$ can be written uniquely as $e \otimes v_{1}+f \otimes v_{2}$, where $v_{1}, v_{2} \in V$ and $\left(v_{1}, v_{2}\right)=1$. So, we can define a surjective morphism $\theta: \Omega_{1} \rightarrow \Omega_{2}$ by $e \otimes v_{1}+f \otimes v_{2} \mapsto v_{1} \wedge v_{2}$. Letting $A$ act on $\Omega_{2}$ trivially, $\theta$ is $L$-equivariant. It is easy to check that the fibres of $\theta$ are $A$-orbits, so that $\theta$ is an orbit map for the action of $A$ on $\Omega_{1}$. Moreover, $\theta$ is separable, so, by [Borel, 6.6], $\left(\Omega_{2}, \theta\right)$ is the quotient of $\Omega_{1}$ by $A$ in the sense of [Borel, 6.3]. Hence, as $\theta$ is $L$-equivariant and $K=L / A$, there is a bijection between closed $L$-orbits in $\Omega_{1}$ and closed $K$-orbits in $\Omega_{2}$. Thus, it is sufficient to show that $L$ has at least two disjoint closed orbits in $\Omega_{1}$.

Let $G$ be a simply connected group of type $E_{7}$ (resp. $E_{8}$ ) if $K=D_{6}$ (resp. $E_{7}$ ). Let $(T, B ; \Sigma, \Pi)$ be a root system for $G$. The maximal rank subgroup obtained by deleting $\alpha_{1}$ (resp. $\left.\alpha_{8}\right)$ from the extended Dynkin diagram of $G$ is of type $A_{1} D_{6}$ (resp. $A_{1} E_{7}$ ), and we identify this with $L$. Let $\left\{H_{\alpha}, X_{\beta} \mid \alpha \in \Pi, \beta \in \Sigma\right\}$ be a Chevalley basis for $\mathfrak{g}=\operatorname{Lie}(G)$. We now claim that we may identify $W$ with the $L$-submodule of $\mathfrak{g}$ spanned by $X_{ \pm \alpha}$ such that $\alpha=a_{1} \alpha_{1}+\cdots+a_{r} \alpha_{r}, a_{1}, \ldots, a_{r} \in \mathbb{Z}$ and $a_{1}= \pm 1$ (resp. $a_{8}= \pm 1$ ). To prove this, observe that $L$ certainly stabilizes this subspace, since each of the root group generators of $L$ stabilizes it. Also, $-\alpha_{1}$ (resp. $\left.-\alpha_{8}\right)$ is equal to the weight $\omega_{1} \otimes \omega_{6}\left(\right.$ resp. $\left.\omega_{1} \otimes \omega_{7}\right)$ when written in terms of fundamental dominant weights for a root system of $L$, so this subspace must be isomorphic to $W$ as an $L$-module.

Now, there is a well defined bilinear form on $\mathfrak{g}$ (defined by reduction modulo $p$ from a scalar multiple of the Killing form on the corresponding Lie algebra over $\mathbb{C})$ satisfying $\left(X_{\alpha}, X_{-\alpha}\right)=1$ for $\alpha \in \Sigma$. Under the identification, this is precisely the bilinear form preserved by $L$ on $W$. Choose $\alpha, \beta \in \Sigma^{+}$such that $X_{\alpha}, X_{\beta} \in W$ and $\alpha+\beta \notin \Sigma$. Let $X_{a, b}=a\left(X_{\alpha}+X_{-\alpha}\right)+b\left(X_{\beta}+X_{-\beta}\right)$ for $a, b \in k$. Consider the infinite set $\Omega_{0}=\left\{X_{a, b} \mid a, b \in k,\left(X_{a, b}, X_{a, b}\right)=1\right\} \subset \Omega_{1}$. Now, as $a, b$ vary, we obtain elements in the fundamental $\mathfrak{s l}_{2} \times \mathfrak{s l}_{2}$ generated by $X_{ \pm \alpha}, X_{ \pm \beta}$ with infinitely many different eigenvalues. Hence, if $p \neq 2$ (when $X_{a, b}$ is semisimple) there are infinitely many closed $G$-orbits intersecting $\Omega_{0}$. If $p=2$, there are infinitely many elements in $\Omega_{0}$ with non-conjugate semisimple parts, so again there are infinitely many $G$-orbits intersecting $\Omega_{0}$ with disjoint closures. Hence, there are infinitely many closed $L$-orbits in $\Omega_{1}$, completing the proof.

2.8. Lemma. There is no dense $H, K$-double coset in $G$ if

$$
(G, H, K, p)=\left(S O(V), B_{6}, N_{1}, 2\right),
$$

where $V \downarrow_{B_{6}}=L_{B_{6}}\left(\omega_{6}\right)$. 
Proof. Let $L=D_{7}$ and $V=L_{D_{7}}\left(\omega_{7}\right)$ with $p=2$. Then, $B_{6}$ is a subgroup of $L$ and $V \downarrow_{B_{6}}=L_{B_{6}}\left(\omega_{6}\right)$. Note that $B_{6}$ preserves a quadratic form $Q$ on $V$, but $L$ does not preserve $Q$. Let $L_{1}$ be the product of $L$ and a one dimensional torus which acts on $V$ by scalars.

We first show that $L_{1}$ has a dense orbit in $V$ with generic stabilizer $G_{2} G_{2}$. To prove this, it is sufficient by dimensions to show that there is $v \in V$ with $\operatorname{stab}_{L}(v)=G_{2} G_{2}$. Now, $V \downarrow_{C_{3} C_{3}}=L_{C_{3}}\left(\omega_{3}\right) \otimes L_{C_{3}}\left(\omega_{3}\right)$. As $G_{2} G_{2}<C_{3} C_{3}$ and $L_{C_{3}}\left(\omega_{3}\right) \downarrow_{G_{2}}$ has a fixed 1-space, it follows that there is some vector $v \in V$ with stabilizer containing $G_{2} G_{2}$. But if the connected stabilizer of $v$ is any larger than $G_{2} G_{2}$ it must contain $G_{2} C_{3}$, which is impossible as $C_{3}$ fixes no vector in $V$.

Now we show that $k[V]^{L} \neq k$. Since $k[V]$ is a unique factorization domain and $L$ has no rational characters, $k(V)^{L}$ is the field of fractions of $k[V]^{L}$. Hence, it is sufficient to show that $k(V)^{L} \neq k$, which by Rosenlicht's theorem [Ros] is equivalent to $L$ having no dense orbit in $V$. But if $L$ has a dense orbit in $V$, then it lies in the dense $L_{1}$-orbit in $V$, and so the generic stabilizer is $G_{2} G_{2}$ by the previous paragraph, which is a contradiction as $\operatorname{dim} D_{7} / G_{2} G_{2}=63 \neq \operatorname{dim} V$.

So, we can find a homogeneous invariant $f \in k[V]^{L}-k$, of degree $d$ say. Since $B_{6}<L$, we just need to show that $f$ is not constant on $\Omega=\{v \in V \mid Q(v)=1\}$, for then $f$ will separate infinitely many closed $B_{6}$-orbits in $\Omega \cong S O(V) / N_{1}$. So, suppose by way of contradiction that $f$ is constant on this set. Consider the regular function $\theta \in k[L]$ defined by $h \mapsto Q\left(h . v_{0}\right)^{d}$ for $h \in L$, where $v_{0} \in V$ is some fixed vector with $Q\left(v_{0}\right)=1$. Since $L$ does not preserve $Q$, we can choose $v_{0}$ so that $\theta$ is not constant on $L$ and $f\left(v_{0}\right) \neq 0$.

Suppose $h$ lies in the dense subset of $L$ defined by the non-vanishing of $\theta$. Then, $\lambda^{2}=Q\left(h . v_{0}\right)$ is non-zero and so $w_{0}=\frac{1}{\lambda} h . v_{0}$ satisfies $Q\left(w_{0}\right)=1$; hence $f\left(w_{0}\right)=$ $f\left(v_{0}\right)$ by assumption. But then $f\left(v_{0}\right)=f\left(h . v_{0}\right)=f\left(\lambda w_{0}\right)=\lambda^{d} f\left(w_{0}\right)=\lambda^{d} f\left(v_{0}\right)$, so $\lambda^{d}=1$ and $\theta(h)=Q\left(h \cdot v_{0}\right)^{d}=Q\left(\lambda w_{0}\right)^{d}=\lambda^{2 d}=1$. This implies that $\theta$ is constant on a dense subset of $L$, hence constant on all of $L$, which is a contradiction.

\section{Proof of Theorem B: tensor embeddings}

In this section, we verify Theorem B for the remaining case in Table 3. We fix some notation throughout the section. Let $U$ and $W$ be non-degenerate symplectic or orthogonal spaces with $\operatorname{dim} U=a, \operatorname{dim} W=b$. Let $H$ be the group $C l(U) \otimes$ $C l(W)$, a central product of the classical groups on $U$ and $W$. Writing $(\cdot, \cdot)$ for the bilinear forms on $U$ and $W, H$ preserves the non-degenerate form on $V=U \otimes W$ defined by $\left(u \otimes w, u^{\prime} \otimes w^{\prime}\right)=\left(u, u^{\prime}\right)\left(w, w^{\prime}\right)$ for $u, u^{\prime} \in U, w, w^{\prime} \in W$, and so it embeds into the corresponding classical group $G=C l(V)$. In characteristic 2, we assume that $H=S p(U), K=S p(W)$; here $H \otimes K$ embeds into $G=S O(V)$. Fix $1 \leq i \leq \frac{1}{2} a b$, where we assume that $i$ is even if $G=S p(V)$ and that $i$ is even or equal to 1 if $(G, p)=(S O(V), 2)$. Let $K=N_{i}<G$.

3.1. Proposition. With the above notation, Theorem $B$ holds for the triple $(G, H, K)$.

We prove Proposition 3.1 with a series of lemmas. Recall that by Lemma 1.4, we just need to show that there are two disjoint closed $H, K$-double cosets in $G$.

3.2. Lemma. Theorem $B$ holds for the triple $(G, H, K)$ if $i=1$.

Proof. Here, $G=S O(V)$. We need to consider the following cases.

(i) $H=S p(U) \otimes S p(W), 2 \leq a \leq b$; 
(ii) $H=S O(U) \otimes S O(W), 3 \leq a \leq b$ and $p \neq 2$.

Pick bases $u_{1}, \ldots, u_{a}$ and $w_{1}, \ldots, w_{b}$ for $U, W$ respectively with respect to which the bilinear forms on $U, W$ correspond to $\left(\begin{array}{cc}0 & I_{n} \\ -I_{n} & 0\end{array}\right)$ if the form is symplectic (where $n=\frac{a}{2}$ or $\frac{b}{2}$ ) or to the identity matrix if the form is orthogonal (possible as $p \neq 2$ ). Denote the matrices corresponding to the forms on $U, W$ by $J_{1}, J_{2}$ respectively. We realise $V$ as the set of $a \times b$ matrices over $k$, with the matrix $m$ corresponding to the vector $\sum m_{s t} u_{s} \otimes w_{t}$ in $V$. If $x=x_{1} \otimes x_{2}$ is an element of $H$ written as a pair of matrices in terms of the chosen bases (so $x_{s}^{T} J_{s} x_{s}=J_{s}$ for $s=1,2$ ), the action of $x$ on $V$ is given by $x: m \mapsto x_{1} m x_{2}^{T}$. Writing $M_{a}(k)$ for the set of $a \times a$ matrices over $k$, define $\theta: V \rightarrow M_{a}(k)$ by $\theta: m \mapsto J_{1} m J_{2} m^{T}$. Then, $\theta: x_{1} m x_{2}^{T} \mapsto x_{1}^{-T}\left(J_{1} m J_{2} m^{T}\right) x_{1}^{T}$, so $H$-orbits in $V$ are send to $H$-conjugacy classes in $M_{a}(k)$.

We first claim that the set $\Omega$ of matrices of the form $m J_{2} m^{T}$ for some $a \times b$ matrix $m$ is just the set of all symmetric matrices in case (ii), or the set of all alternating matrices in case (i). As $b \geq a$, it is sufficient to prove the claim in the case $b=a$. Any symmetric (resp. alternating) matrix $F$ can be regarded as the matrix for a symmetric (resp. alternating) bilinear form on $V$. Any such form can be reduced to a canonical form by change of basis, which corresponds to the matrix operation $F \mapsto m F m^{T}$ for some matrix $m$. Since $\Omega$ is closed under this operation, one just needs to check that the matrices corresponding to one's favourite canonical form for symmetric (resp. alternating) bilinear forms lie in $\Omega$, which is straightforward. Hence, the image of $\theta$ is the set of all symmetric matrices in case (ii) or

$$
\left\{\left(\begin{array}{cc}
m_{1} & m_{2} \\
m_{3} & m_{1}^{T}
\end{array}\right) \mid \text { for all } m_{1}, m_{2}, m_{3} \in M_{a}(k) \text { with } m_{2}, m_{3} \text { alternating }\right\}
$$

in case (i).

Now, if $p \neq 2$, we can realise $K$ as the stabilizer of a vector $v \in V$ with $(v, v)=1$. Then, $\theta(G . v)$ is just the set of all matrices in $\theta(V)$ of trace 1 . We now claim that there are at least two disjoint closed $H$-orbits in $\theta(G . v)$ if $a>2$ (the case $a=2$ is a factorization by [LSS]). The result will then follow with an application of Lemma 1.4, for on taking pre-images, we see that there are at least two disjoint closed $H$-orbits in $G . v \cong G / K$. To prove the claim, note that the action of $H$ on $M_{a}(k)$ is conjugation. There are clearly non-conjugate diagonal matrices in $\theta(G . v)$ for the action of all of $G L_{a}(k)$ on $M_{a}(k)$, provided $a>2$. Each $G L_{a}(k)$-conjugacy class of a diagonal matrix is closed, hence always contains at least one closed $H$ conjugacy class. Hence, $H$ has disjoint closed orbits in $\theta(G . v)$, as required.

If $p=2$, one needs to argue further. The quadratic form preserved by $G$ on $V$ is given explicitly, in terms of the basis, by $Q\left(u_{s} \otimes w_{t}\right)=0$ for all $s, t$. Given this, it is not hard to exhibit vectors $v, v^{\prime} \in V$ with $Q(v)=1=Q\left(v^{\prime}\right)$ such that $\theta(v)$ and $\theta\left(v^{\prime}\right)$ are non-conjugate diagonal matrices, and then the preceding argument completes the proof.

Now assume $i>1$. Some of the arguments here break down for the case of $N_{2}$ in $S O(V)$ (which is a Levi subgroup), so we shall divide this into five cases:

(i) $H=S O(U) \otimes S O(W), p \neq 2$ and $3 \leq a \leq b$;

(ii) $H=S p(U) \otimes S O(W), p \neq 2$ and either $2 \leq a \leq b, 3 \leq b$ or $i=2,3 \leq b<a$;

(iii) $H=S O(U) \otimes S p(W), p \neq 2$ and $3 \leq a<b$ with $i \neq 2$;

(iv) $H=S p(U) \otimes S p(W), 2 \leq a \leq b, 4 \leq b$ and $i \neq 2$;

(v) $H=S p(U) \otimes S p(W), 2 \leq a \leq b, 4 \leq b$ and $i=2$. 
3.3. Lemma. There is no dense $H, K$-double coset in $G$ if $i>b$.

Proof. We show that in this case, $\operatorname{dim} H+\operatorname{dim} K<\operatorname{dim} G$. This is just an elementary (but messy) manipulation of inequalities. Note that $a \leq b$ (the second possibility in (ii) does not occur as $i>b$ ). The inequality $\operatorname{dim} H+\operatorname{dim} K \geq \operatorname{dim} G$ is then equivalent to

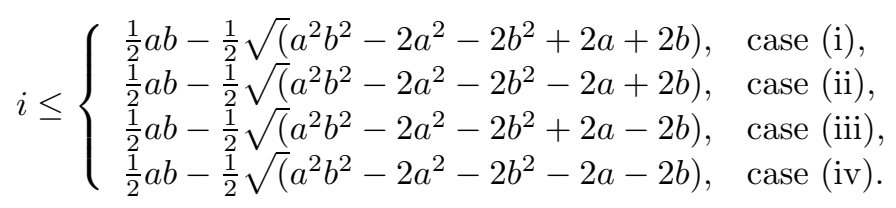

Hence, in all cases, it is sufficient to show that

$$
b \geq \frac{1}{2} a b-\frac{1}{2} \sqrt{\left(a^{2} b^{2}-2 a^{2}-2 b^{2}-2 a-2 b\right) .}
$$

First, if $a=b$, then this inequality reduces to $\left.b \geq \frac{1}{2} b^{2}-\frac{1}{2} \sqrt{(} b^{4}-4 b^{2}-4 b\right)$, which is easily seen to be true when $b \geq 3$. Otherwise, $a<b$. Then, $a(a+1) \leq b(b-1)$. So, $0 \leq 2 b^{2}-2 a^{2}-2 b-2 a$. Hence, $a^{2} b^{2}-4 b^{2} \leq a^{2} b^{2}-2 b^{2}-2 a^{2}-2 b-2 a$. Making this substitution in $(\dagger)$, the inequality is then easy to verify.

Thus, from now on, we assume that we are in one of cases (i)-(iv) - we will treat case (v) separately in Lemma 3.7 - and that $1<i \leq b$. We now fix standard bases for $U, W$. Let $A=\left[\frac{a}{2}\right], B=\left[\frac{b}{2}\right]$. Let $e_{1}, \ldots, e_{A}, f_{1}, \ldots, f_{A}$, together with $d$ if $a$ is odd, be a basis for $U$ such that

$$
\begin{aligned}
& \left(e_{s}, e_{t}\right)=\left(f_{s}, f_{t}\right)=0, \quad\left(e_{s}, f_{t}\right)=\delta_{s t}, \\
& \left(d, e_{s}\right)=\left(d, f_{s}\right)=0, \quad(d, d)=1
\end{aligned}
$$

for all $s, t$. Similarly, let $e_{1}^{\prime}, \ldots, e_{B}^{\prime}, f_{1}^{\prime}, \ldots, f_{B}^{\prime}$, together with $d^{\prime}$ if $b$ is odd, be a basis for $W$.

Let $V_{1}=\left\langle v_{1}, \ldots, v_{i}\right\rangle$ be a non-degenerate $i$-subspace of $V$, where $v_{1}, \ldots, v_{i}$ are chosen so that the determinant of the $i \times i$ matrix with st-entry equal to $\left(v_{s}, v_{t}\right)$ is 1. Let $K=\operatorname{stab}_{G}\left(V_{1}\right)$; equivalently, $K=\operatorname{stab}_{G}\left(v_{1} \wedge \cdots \wedge v_{i}\right)$, the stabilizer of a vector in $\bigwedge^{i} V$. The $G$-orbit containing $v_{1} \wedge \cdots \wedge v_{i}$ is just

$$
\left\{w_{1} \wedge \cdots \wedge w_{i} \mid w_{s} \in V, \operatorname{det}\left(w_{s}, w_{t}\right)=1\right\} .
$$

Define a linear map $\theta: \bigwedge^{i} V \rightarrow S^{i} U \otimes \bigwedge^{i} W$ by $\theta:\left(u_{1} \otimes w_{1}\right) \wedge \cdots \wedge\left(u_{i} \otimes w_{i}\right) \mapsto$ $\left(u_{1} \cdot \ldots \cdot u_{i}\right) \otimes\left(w_{1} \wedge \cdots \wedge w_{i}\right)$ for $u_{s} \in U, w_{t} \in W$. Then, $\theta$ is $H$-equivariant, so the morphism $\bar{\theta}: G \rightarrow S^{i} U \otimes \bigwedge^{i} W$, defined by $\bar{\theta}: g \mapsto \theta\left(g \cdot v_{1} \wedge \cdots \wedge v_{i}\right)$ for $g \in G$, sends $H, K$-double cosets in $G$ to $H$-orbits in $S^{i} U \otimes \bigwedge^{i} W$. It is sufficient to show that there are two $H$-orbits in $\bar{\theta}(G)$ with disjoint closures, since their pre-images then contain two disjoint closed $H, K$-double cosets in $G$, and Lemma 1.4 then implies Theorem B holds in this case. The first closed orbit is easy to find (this is the reason we chose the conditions in cases (i)-(iv) with some care).

3.4. Lemma. In cases (i)-(iv), $0 \in \bar{\theta}(G)$.

Proof. If $i \geq 4$, we may choose $V_{1}$ such that $v_{1}=e_{1} \otimes e_{1}^{\prime}, v_{2}=f_{1} \otimes f_{1}^{\prime}, v_{3}=$ $e_{1} \otimes f_{1}^{\prime}, v_{4}=f_{1} \otimes e_{1}^{\prime}$. Then, $\bar{\theta}(1)=\theta\left(v_{1} \wedge \cdots \wedge v_{i}\right)$, which is zero as every term in the expression will contain $e_{1}^{\prime} \wedge e_{1}^{\prime}$. If $i=3$, so $p \neq 2$, we may choose $V_{1}$ so that $v_{1}=e_{1} \otimes e_{1}^{\prime}, v_{2}=f_{1} \otimes f_{1}^{\prime}, v_{3}=\frac{1}{\sqrt{2}}\left(e_{1} \otimes f_{1}^{\prime}+f_{1} \otimes e_{1}^{\prime}\right)$, whence it is easily checked 
that $\bar{\theta}(1)=0$. Finally, if $i=2$ then $C l(W)=S O(W)$ and $p \neq 2$. Let $w$ be a nonsingular vector in $W$. Then, we may choose $V_{1}$ so that $v_{1}=e_{1} \otimes w, v_{2}=f_{1} \otimes w$, and $\bar{\theta}(1)=0$.

Thus, it is sufficient in cases (i)-(iv) to show that there is $v \in \bar{\theta}(G)$ with $0 \notin \overline{H . v}$ (except for case (v)). Let $T$ be the maximal torus of $H$ that acts diagonally on the standard bases for $U, W$ chosen above.

3.5. Lemma. In cases (i)-(iv) with $i$ even, there is no dense $H, K$-double coset in G.

Proof. Let $V_{2}$ be the non-degenerate $k$-space spanned by $e_{1} \otimes e_{1}^{\prime}, f_{1} \otimes f_{1}^{\prime}, \ldots, e_{1} \otimes$ $e_{j}^{\prime}, f_{1} \otimes f_{j}^{\prime}$, where $j=\frac{i}{2}$. Then, the image of this in $\bar{\theta}(G)$ is

$$
v=\left(e_{1} . f_{1}\right)^{j} \otimes\left(e_{1}^{\prime} \wedge f_{1}^{\prime} \wedge \cdots \wedge e_{j}^{\prime} \wedge f_{j}^{\prime}\right)
$$

This is a non-zero vector of $S^{i} U \otimes \bigwedge^{i} W$ which is fixed by T. Hence, by (1.6), H.v is closed, and so $\overline{H . v}$ is disjoint from 0 , as required.

To treat the remaining cases, we have to do rather more work. Suppose that $U_{1}$ and $W_{1}$ are $H_{1}$-modules for some algebraic group $H_{1}$, and that $H_{1}$ preserves a bilinear form $\langle\cdot, \cdot\rangle$ on $W_{1}$. We now define an $H_{1}$-equivariant morphism $U_{1} \otimes W_{1} \rightarrow$ $U_{1} \otimes U_{1}$, which we shall use a number of times in the remainder of the section. Fix bases $u_{1}, \ldots, u_{N}$ and $w_{1}, \ldots, w_{M}$ for $U_{1}$ and $W_{1}$ respectively. Define the morphism $\phi: U_{1} \otimes W_{1} \rightarrow U_{1} \otimes U_{1}$ by

$$
\phi: \sum_{s, t} a_{s t} u_{s} \otimes w_{t} \mapsto \sum_{s, t, s^{\prime}, t^{\prime}} a_{s t} a_{s^{\prime} t^{\prime}}\left\langle w_{t}, w_{t^{\prime}}\right\rangle u_{s} \otimes u_{s^{\prime}},
$$

where $s, s^{\prime}$ sum between 1 and $N$ and $t, t^{\prime}$ sum between 1 and $M$. This is certainly a morphism. A routine (if slightly gruesome) check shows that the definition of $\phi$ does not depend on the choice of basis. Using this observation, it is easy to check that $\phi$ is $H$-equivariant.

3.6. Lemma. In cases (i)-(iv) with $i$ odd, there is no dense $H, K$-double coset in $G$.

Proof. As $i$ is odd and $i \neq 1$, either $H=S O(U) \otimes S O(W)$ or $H=S p(U) \otimes S p(W)$, and $p \neq 2$. Let $V_{2}$ be the non-degenerate $i$-space spanned by $e_{1} \otimes e_{1}^{\prime}, f_{1} \otimes f_{1}^{\prime}, \ldots, e_{1} \otimes$ $e_{j}^{\prime}, f_{1} \otimes f_{j}^{\prime}, e_{1} \otimes e_{j+1}^{\prime}+f_{1} \otimes f_{j+1}^{\prime}$ where $j=\left[\frac{i}{2}\right]$. Its image in $S^{i} U \otimes \bigwedge^{i} W$ is $v=e_{1}^{j} . f_{1}^{j+1} \otimes e_{1}^{\prime} \wedge f_{1}^{\prime} \wedge \cdots \wedge e_{j}^{\prime} \wedge f_{j}^{\prime} \wedge f_{j+1}^{\prime}+e_{1}^{j+1} . f_{1}^{j} \otimes e_{1}^{\prime} \wedge f_{1}^{\prime} \wedge \cdots \wedge e_{j}^{\prime} \wedge f_{j}^{\prime} \wedge e_{j+1}^{\prime}$. We show that $0 \notin \overline{H . v}$, which will complete the proof.

Let $N=\operatorname{dim} S^{i} U, M=\operatorname{dim} \bigwedge^{i} W$, and let $u_{1}, \ldots, u_{N}\left(\right.$ resp. $\left.w_{1}, \ldots, w_{M}\right)$ be bases for $S^{i} U$ (resp. $\bigwedge^{i} W$ ) such that $u_{1}=e_{1}^{j+1} \cdot f_{1}^{j}, u_{2}=e_{1}^{j} \cdot f_{1}^{j+1}$ and $w_{1}=$ $e_{1}^{\prime} \wedge f_{1}^{\prime} \wedge \cdots \wedge e_{j}^{\prime} \wedge f_{j}^{\prime} \wedge e_{j+1}^{\prime}, w_{2}=e_{1}^{\prime} \wedge f_{1}^{\prime} \wedge \cdots \wedge e_{j}^{\prime} \wedge f_{j}^{\prime} \wedge f_{j+1}^{\prime}$.

Now, $H$ preserves the canonical form on $\wedge^{i} W$, defined by

$$
\left\langle a_{1} \wedge \cdots \wedge a_{i}, b_{1} \wedge \cdots \wedge b_{i}\right\rangle=\sum_{\sigma \in S_{i}} \varepsilon(\sigma)\left(a_{1}, b_{\sigma 1}\right) \cdot \cdots \cdot\left(a_{i}, b_{\sigma i}\right)
$$

for $a_{s}, b_{t} \in W$. We now construct an $H$-equivariant morphism $\phi: S^{i} U \otimes \bigwedge^{i} W \rightarrow$ $S^{i} U \otimes S^{i} U$ as in (*) (taking $\left.\left(H_{1}, U_{1}, W_{1}\right)=\left(H, S^{i} U, \bigwedge^{i} W\right)\right)$. Explicitly,

$$
\phi: \sum_{s, t} a_{s t} u_{s} \otimes w_{t} \mapsto \sum_{s, t, s^{\prime}, t^{\prime}} a_{s t} a_{s^{\prime} t^{\prime}}\left\langle w_{t}, w_{t^{\prime}}\right\rangle u_{s} \otimes u_{s^{\prime}} .
$$


Now, one computes the image of $v=u_{1} \otimes w_{1}+u_{2} \otimes w_{2}$, to show that $\phi(v)=$ $(-1)^{j}\left(u_{1} \otimes u_{2}+u_{2} \otimes u_{1}\right)$ or $u_{1} \otimes u_{2}-u_{2} \otimes u_{1}$ according to whether $H=S O(U) \otimes$ $S O(W)$ or $S p(U) \otimes S p(W)$ respectively. Now, $u_{1} \otimes u_{2}=e_{1}^{j+1} \cdot f_{1}^{j} \otimes e_{1}^{j} \cdot f_{1}^{j+1}$, which is fixed by $T$, and similarly for $u_{2} \otimes u_{1}$. Hence, $\phi(v)$ is fixed by $T$, so its $H$-orbit is closed by Lemma 1.6, and disjoint from 0. Hence, the pre-image of $H . \phi(v)=\phi(H . v)$ will be closed and disjoint from zero, and contains H.v. But this shows that $0 \notin \overline{H . v}$, as required.

It remains to treat case (v). Here, we have to proceed slightly differently.

3.7. Lemma. There is no dense $H, K$-double coset in $G$ in case (v).

Proof. Let $Q$ be the quadratic form associated to $(\cdot, \cdot)$ on $V$ preserved by $G$. Let $\Omega=\left\{v_{1} \otimes v_{2} \in V \otimes V \mid\left(v_{1}, v_{2}\right)=1, Q\left(v_{1}\right)=0=Q\left(v_{2}\right)\right\}$. Then, $G$ acts transitively on $\Omega$ and $K$ is the stabilizer of a vector in $\Omega$, so we just need to show $H$ has at least two disjoint closed orbits in $\Omega$. Let $\langle\cdot, \cdot\rangle$ be the $H$-invariant bilinear form on $W \otimes W$ defined by $\left\langle w_{1} \otimes w_{2}, w_{1}^{\prime} \otimes w_{2}^{\prime}\right\rangle=\left(w_{1}, w_{2}^{\prime}\right)\left(w_{2}, w_{1}^{\prime}\right)$. Now, we define an $H$-equivariant morphism $V \otimes V \rightarrow S^{2}(U \otimes U)$ by composition,

$$
\theta: V \otimes V \longrightarrow U \otimes U \otimes W \otimes W \stackrel{\phi}{\longrightarrow} U \otimes U \otimes U \otimes U \longrightarrow S^{2}(U \otimes U),
$$

where the first and last maps are canonical and $\phi$ is as defined in $(*)$ with

$$
\left(H_{1}, U_{1}, W_{1}\right)=(H, U \otimes U, W \otimes W) .
$$

Compute the image of $\left(\eta e_{1} \otimes e_{1}^{\prime}+(1-\eta) f_{1} \otimes f_{2}^{\prime}\right) \otimes\left(f_{1} \otimes f_{1}^{\prime}+e_{1} \otimes e_{2}^{\prime}\right) \in \Omega$. It is

$$
-\eta^{2}\left(e_{1} \otimes f_{1}\right)^{2}+2 \eta(1-\eta) e_{1} \otimes e_{1} . f_{1} \otimes f_{1}-(1-\eta)^{2}\left(f_{1} \otimes e_{1}\right)^{2} .
$$

In particular, the image consists of infinitely many elements of weight zero as $\eta$ varies. Hence, there are infinitely many closed $H$-orbits in $\theta(\Omega)$ by Lemma 1.9 and (1.6), and the conclusion follows by Lemma 1.4.

This completes the proof of Proposition 3.1.

Finally, we consider a case in $S L(V)$, using the same argument as for Lemma 3.7.

3.8. Lemma. There is no dense $H, K$-double coset in $G$ if

$$
(G, H, K)=\left(S L_{a b}, S L_{a} \otimes S L_{b}, G L_{a b-1}\right) .
$$

Proof. Let $V=U \otimes W$ with $\operatorname{dim} V=a b, \operatorname{dim} U=a, \operatorname{dim} W=b$, and let $G=$ $S L(V), H=S L(U) \otimes S L(W)$. Fix bases $u_{1}, \ldots, u_{a}$ for $U$ and $w_{1}, \ldots, w_{b}$ for $W$, and let $\bar{u}_{1}, \ldots, \bar{u}_{a}$ (resp. $\bar{w}_{1}, \ldots, \bar{w}_{b}$ ) be the corresponding dual basis for $U^{*}$ (resp. $\left.W^{*}\right)$.

Note that $K$ is the stabilizer of an element $v_{0} \otimes \bar{v}_{0} \in V \otimes V^{*}$ where $\bar{v}_{0}\left(v_{0}\right)=1$. Hence, define $\Omega=\left\{v \otimes \bar{v} \in V \otimes V^{*} \mid \bar{v}(v)=1\right\}$, the $G$-orbit of $v_{0} \otimes \bar{v}_{0}$. We need to prove that $H$ has at least two disjoint closed orbits in $\Omega$, by Lemma 1.4.

Let $\langle.,$.$\rangle be the H$-equivariant bilinear form on $W \otimes W^{*}$ defined by

$$
\left\langle w_{s} \otimes \bar{w}_{t}, w_{s^{\prime}} \otimes \bar{w}_{t^{\prime}}\right\rangle=\bar{w}_{t}\left(w_{s^{\prime}}\right) \bar{w}_{t^{\prime}}\left(w_{s}\right)
$$

for $1 \leq s, s^{\prime}, t, t^{\prime} \leq b$. Now, $V \otimes V^{*}$ is just $U \otimes W \otimes U^{*} \otimes W^{*}$. Define an $H$-equivariant morphism $\theta: V \otimes V^{*} \rightarrow S^{2}\left(U \otimes U^{*}\right)$ by the composition

$$
V \otimes V^{*} \longrightarrow U \otimes U^{*} \otimes W \otimes W^{*} \stackrel{\phi}{\longrightarrow} U \otimes U^{*} \otimes U \otimes U^{*} \longrightarrow S^{2}\left(U \otimes U^{*}\right),
$$

where the first and last maps are canonical, and $\phi$ is as defined in $(*)$ with

$$
\left(H_{1}, U_{1}, W_{1}\right)=\left(H, U \otimes U^{*}, W \otimes W^{*}\right) .
$$


Compute the image of $\left(\eta u_{1} \otimes w_{1}+(1-\eta) u_{2} \otimes w_{2}\right) \otimes\left(\bar{u}_{1} \otimes \bar{w}_{1}+\bar{u}_{2} \otimes \bar{w}_{2}\right) \in \Omega$. It is

$$
\eta^{2}\left(u_{1} \otimes \bar{u}_{1}\right)^{2}+2 \eta(1-\eta)\left(u_{1} \otimes \bar{u}_{2} \otimes u_{2} \otimes \bar{u}_{1}\right)+(1-\eta)^{2}\left(u_{2} \otimes \bar{u}_{2}\right)^{2} .
$$

The important thing is that the image is of weight zero with respect to the maximal torus $T$ of $H$ that acts diagonally on the basis, and as $\eta$ varies we obtain infinitely many distinct elements. Hence, there are infinitely many closed $H$-orbits in $\theta(\Omega)$ by Lemma 1.9 and (1.6).

\section{Proof of Theorem B: Levi factors}

We now consider Theorem B when one or both of $H$ or $K$ is a Levi factor. So, let $G$ be a connected reductive algebraic group. We fix a root system $(T, B ; \Sigma, \Pi)$ for $G$ for the remainder of this section. Let $P=P_{J}$ be the standard parabolic subgroup of $G$ corresponding to the subset $J$ of $I=\{1, \ldots, n\}$. So, $P=\left\langle B, U_{-\alpha_{j}} \mid j \in J\right\rangle$, and $L=\left\langle T, U_{ \pm \alpha_{j}} \mid j \in J\right\rangle$ is a Levi factor of $P$. Let $W_{L}=N_{L}(T) / T$ be the Weyl group of $L$, a subgroup of $W$.

4.1. Lemma ([B2, Lemma 3.2]). Let $H$ be a connected reductive subgroup of $G$ with maximal torus $S \leq T$; let $W_{H}=N_{H}(S) / S$ be the Weyl group of $H$. Suppose $N_{H}(S)=N_{H}(T)$, so that $W_{H}$ can be identified with a subgroup of $W$. If there is a dense $H, L$-double coset in $G$, then $W=W_{H} W_{L}$ is a factorization of $W$.

The condition $N_{H}(S)=N_{H}(T)$ in Lemma 4.1 is obviously satisfied if $H$ is of maximal rank, for then we can take $S=T$. As an immediate application, we have the following:

4.2. Corollary. Let $L_{J}$ and $L_{J^{\prime}}$ be standard Levi factors corresponding to proper subsets $J, J^{\prime} \subset I$. Then, there is no dense $L_{J}, L_{J^{\prime}}$-double coset in $G$.

Proof. Let $W_{J}, W_{J^{\prime}}$ be the corresponding parabolic subgroups of $W$. By Lemma 4.1, we just need to show that $W \neq W_{J} W_{J^{\prime}}$, which is well known.

To apply Lemma 4.1 to subgroups $H$ which are not of maximal rank, we need to verify the condition $N_{H}(S)=N_{H}(T)$. We now consider this problem. We write $\mathfrak{t}$ and $\mathfrak{g}$ for the Lie algebras of $T$ and $G$ respectively. We shall need the next, known lemma:

4.3. Lemma ([B2, Lemma 3.6]). Suppose $G$ is simple, simply connected and $(G, p)$ is not $\left(C_{n}, 2\right)$ for any $n \geq 1$. Then, $N_{G}(\mathfrak{t})=N_{G}(T)$.

4.4. Corollary. Let $G$ be as in the lemma, and let $H$ be a connected reductive subgroup with maximal torus $S<T$. Suppose the zero weight space of $\mathfrak{g}$ relative to $S$ is equal to $\mathfrak{t}$. Then, $N_{H}(S)=N_{H}(T)$.

Proof. By assumption, $N_{H}(S)$ normalizes $\mathfrak{g}$. Hence, by Lemma 4.3, $N_{H}(S) \leq$ $H \cap N_{G}(\mathfrak{t})=H \cap N_{G}(T)=N_{H}(T)$. Conversely, let $n \in N_{H}(T)$ and let $s \in S=$ $T \cap H$. Then, $n s n^{-1} \in T \cap H=S$, so $n$ normalizes $S$.

4.5. Lemma. Let $G=C l(V)$, and let $H<G$ be a connected reductive subgroup with maximal torus $S<T$. Then, the zero weight space of $\mathfrak{g}$ relative to $S$ equals $\mathfrak{t}$ if and only if the following hold:

(i) for every $\mu \in X(S), \operatorname{dim}\left(V \downarrow_{S}\right)_{\mu} \leq 1$;

(ii) if $G=C_{n}$ or $B_{n}(p=2)$, then $\left(V \downarrow_{S}\right)_{0}=0$. 
TABle 4. Case list involving a Levi factor

\begin{tabular}{|l|l|l|l|c|}
\hline & $p$ & $H$ & $K$ & Ref \\
\hline \hline$S L(V)$ & & $S L(U) \otimes S L(W), V=U \otimes W$ & $L_{i}$ & $4.7,3.8$ \\
$S L(V)$ & & $S p(V)$ & $L_{i}$ & 4.7 \\
$S L(V)$ & $p \neq 2$ & $S O(V)$ & $L_{i}$ & $4.7,4.8$ \\
\hline$S O(V)$ & & $C l(U) \otimes C l(W), V=U \otimes W$ & $N_{2}$ & 3.1 \\
$S p_{2 n}$ & $p \neq 2$ & $N_{i}$ & $G L_{n}$ & 4.9 \\
$S O_{2 n}$ & & $N_{i}$ & $G L_{n}$ & $4.7,4.9$ \\
\hline$S O_{7}$ & $p \neq 2$ & $G_{2}, V \downarrow_{H}=L_{H}\left(\omega_{1}\right)$ & $N_{2}$ & {$[\mathrm{LSS}]$} \\
$S O_{8}$ & & $B_{3}, V \downarrow_{H}=L_{H}\left(\omega_{1}\right)$ & $N_{2}$ & {$[\mathrm{LSS}]$} \\
$S O_{8}$ & & $N_{1}$ & $N_{2}$ & 2.2 \\
$S O_{16}$ & & $B_{4}$ & $N_{2}$ & 4.11 \\
$S O_{26-\delta p, 3}$ & & $F_{4}, V \downarrow_{H}=L_{H}\left(\omega_{4}\right)$ & $N_{2}$ & 4.14 \\
$S O_{32}$ & $p=2$ & $D_{6}, V \downarrow_{H}=L_{H}\left(\omega_{6}\right)$ & $N_{2}$ & 2.7 \\
$S O_{56}$ & $p=2$ & $E_{7}, V \downarrow_{H}=L_{H}\left(\omega_{7}\right)$ & $N_{2}$ & 2.7 \\
\hline
\end{tabular}

Proof. We prove this for the case $G=S p(V)$; other types of $G$ are similar. Let $e_{1}, \ldots, e_{n}, f_{n}, \ldots, f_{1}$ be a symplectic basis for $V$, where $\left(e_{i}, e_{j}\right)=0=\left(f_{i}, f_{j}\right)$ and $\left(e_{i}, f_{j}\right)=\delta_{i j}$. Let $T$ be the maximal torus of $G$ which acts diagonally on this basis, so elements of $T$ have the form $t=\operatorname{diag}\left(t_{1}, \ldots, t_{n}, t_{n}^{-1}, \ldots, t_{1}^{-1}\right)$ when written with respect to the basis. Let $\varepsilon_{i}$ be the character of $T$ sending $t \mapsto t_{i}$ for $1 \leq i \leq n$. Then, the weights of $e_{i}, f_{i}$ are $\varepsilon_{i},-\varepsilon_{i}$ respectively. Let $\mu_{i}$ be the restriction of $\varepsilon_{i}$ to $S$. The weights of $\mathfrak{g}$ are $\left\{0^{n}, \pm 2 \varepsilon_{i}, \pm \varepsilon_{i} \pm \varepsilon_{j} \mid 1 \leq i<j \leq n\right\}$. We therefore require the weights $2 \mu_{i}$ and $\mu_{i} \pm \mu_{j}$ to $S$ to be non-zero for each $1 \leq i<j \leq n$. This clearly holds if and only if no $\mu_{i}=0$, so $\left(V \downarrow_{S}\right)_{0}=0$, and no $\mu_{i}= \pm \mu_{j}$, so $\operatorname{dim}\left(V \downarrow_{S}\right)_{\mu} \leq 1$ for all $\mu \in X(S)$.

Now we apply these results to the remaining cases in the proof of Theorem B, when one of $H, K$ is a Levi factor. We begin by obtaining a case list as before.

4.6. Proposition. Let $G$ be simple and $H, K \in \mathcal{M}(G)$, with $K$ a Levi factor. To prove $B$ for the triple $(G, H, K)$ it is sufficient to show that it holds for the $(G, H, K)$ in Table 4. (In the table we reference the lemma in which we treat these subgroups.)

Proof. The possibilities for $(G, K)$ are easy to compute. Moreover, it is sufficient to list the possibilities for $H, K$ up to graph automorphisms of $G$. Hence, $(G, K)=$ $\left(A_{n}, L_{i}\right)\left(1 \leq i \leq \frac{n}{2}\right),\left(B_{n}, L_{1}\right),\left(C_{n}, L_{n}\right),\left(D_{n}, L_{1}\right)$ or $\left(D_{n}, L_{n}\right)(n>4)$. Next, note that we can exclude $(G, K, p)=\left(C_{n}, L_{n}, 2\right)$, as then $L_{n}$ lies in a subgroup $D_{n}$ so that $K \notin \mathcal{M}(G)$. Then, by Corollary $4.2, H$ is not a Levi factor, so $H$ is a reductive maximal connected subgroup of $G$ with $\operatorname{dim} H \geq \operatorname{dim} G / K$. Now the possibilities can be listed, copying the proof of Proposition 2.1.

We begin the proof of these cases by considering factorizations in the Weyl group, applying Lemma 4.1 when possibile. 
4.7. Lemma. Theorem $B$ holds if

$$
\begin{aligned}
& (G, H, K)=\left(S L(V), S L(U) \otimes S L(W), L_{i}\right)(i \neq 1), \\
& (G, H, K)=\left(S O_{2 n}, N_{i}, G L_{n}\right)(i \text { even }) \\
& (G, H, K)=\left(S L(V), S p(V), L_{i}\right) \\
& (G, H, K)=\left(S L(V), S O(V), L_{i}\right)(p \neq 2)
\end{aligned}
$$

Proof. First observe that for each case the condition in Lemma 4.5 is satisfied. Thus, we may apply Corollary 4.4 to deduce that the condition $N_{H}(S)=N_{H}(T)$ is satisfied in every case. Thus, it suffices to show that $W \neq W_{H} W_{L}$, where $W_{H}$ and $W_{L}$ are the Weyl groups of $H$ and $L=K$ respectively by Lemma 4.1. We consider this for each case in turn.

(i) $(G, H, K)=\left(S L(V), S L(U) \otimes S L(W), L_{i}\right)(i \neq 1)$. Let $n=\operatorname{dim} G, r=n-1$. By applying a graph automorphism, we may assume $i \leq \frac{r}{2}$. We shall show that $W \neq W_{H} W_{L}$.

Choose bases $u_{1}, \ldots, u_{a}$ and $w_{1}, \ldots, w_{b}$ for $U, W$ respectively, and assume $a \leq b$. An easy dimension argument shows that we may assume $i \leq b$. Let $T_{U} \otimes T_{W}$ be the corresponding maximal torus of $H$ that acts diagonally on these basis elements. Let $v_{1}, \ldots, v_{a b}$ be the basis for $U \otimes W$ such that $v_{(i-1) b+j}=u_{i} \otimes w_{j}$, where $1 \leq i \leq$ $a, 1 \leq j \leq b$. Let $T$ be the maximal torus of $G$ that acts diagonally on this basis, so $T_{U} \otimes T_{W}<T$. Write $\varepsilon_{i}$ for the element of $X(T)$ that sends $t \mapsto t_{i}$ when $t \in T$ is written as $\operatorname{diag}\left(t_{1}, \ldots, t_{a b}\right)$ with respect to the basis $v_{1}, \ldots, v_{a b}$. Let $\mu_{i j}$ be the element of $X\left(T_{U} \otimes T_{W}\right)$ obtained by restricting $\varepsilon_{(i-1) b+j}$.

Now, by the proof of [B2, Lemma 3.2], $W_{L}$ is the stabilizer of $\omega_{i}$ in $X(T)$, and $\omega_{i}=\varepsilon_{1}+\cdots+\varepsilon_{i}$. Hence, $W \cdot \omega_{i}=\left\{\varepsilon_{j_{1}}+\cdots+\varepsilon_{j_{i}} \mid 1 \leq j_{1}<\cdots<j_{i} \leq a b\right\}$ is of order $a\left(\begin{array}{l}b \\ i\end{array}\right)$. On the other hand, we are assuming $i \leq b$, so $\omega_{i} \downarrow_{T_{U} \otimes T_{W}}=\mu_{11}+\cdots+\mu_{1 i}$. So, $W_{H} \cdot \omega_{i}=\left\{\mu_{j k_{1}}+\cdots+\mu_{j k_{i}} \mid 1 \leq j \leq a, 1 \leq k_{1}<\cdots<k_{i} \leq b\right\}$ of order $a\left(\begin{array}{l}b \\ i\end{array}\right)$. Hence, $W_{H} \cdot \omega_{i} \neq W \cdot \omega_{i}$ unless $i=1$, and the result follows.

(ii) $(G, H, K)=\left(S O_{2 n}, N_{2 i}, G L_{n}\right)$. Again $W \neq W_{H} W_{L}$. Let $e_{1}, \ldots, e_{n}, f_{n}, \ldots, f_{1}$ be a basis for $V$ such that $\left(e_{s}, e_{t}\right)=0=\left(f_{s}, f_{t}\right),\left(e_{s}, f_{t}\right)=\delta_{s t}$ and $Q\left(e_{s}\right)=Q\left(f_{t}\right)=$ 0 , where $Q$ is the associated quadratic form if $p=2$. Let $\varepsilon_{1}, \ldots, \varepsilon_{n}$ be the corresponding weights of the maximal torus $T$ that acts diagonally on this basis. Then, $W_{L}$ is the stabilizer of $\omega_{n}=\frac{1}{2}\left(\varepsilon_{1}+\cdots+\varepsilon_{n}\right)$ in $X(T)$. Also, $W$ acts on $\pm \varepsilon_{1}, \ldots, \pm \varepsilon_{n}$ as all permutations of $1, \ldots, n$ and all sign changes of even signature. Hence, $\left|W \cdot \omega_{n}\right|=2^{n-1}$. On the other hand, $\left|W_{H} \cdot \omega_{n}\right|=2^{i-1} 2^{n-i-1}=2^{n-2}$. Hence, $W \neq W_{H} W_{L}$.

(iii) $(G, H, K)=\left(S L_{2 n}, S p_{2 n}, L_{i}\right)(i \neq 1)$. If $i=1$, this is a factorization by [LSS]. So, suppose $1<i \leq n$. Let $T$ be a maximal torus of $G$ and $\varepsilon_{1}, \ldots, \varepsilon_{2 n}$ be the weights of $T$ as in (i). Then, we may choose $T_{H}$ and weights $\mu_{1}, \ldots, \mu_{n}$ as usual such that $\varepsilon_{i} \downarrow_{T_{H}}=-\varepsilon_{n+i} \downarrow_{T_{H}}=\mu_{i}$ for $1 \leq i \leq n$. Then, $L_{i}$ is the stabilizer of $\omega_{i}=\varepsilon_{1}+\cdots+\varepsilon_{i}$. Hence, $\left|W \cdot \omega_{i}\right|=\left(\begin{array}{c}2 n \\ i\end{array}\right)$ as in (i). Now, $W_{H}$ acts as all permutations and sign changes on $\pm \mu_{1}, \ldots, \pm \mu_{n}$. Hence, $\left|W_{H} \cdot \omega_{i}\right|=2^{i}\left(\begin{array}{c}n \\ i\end{array}\right)$. Hence, $W \neq W_{H} W_{L}$ provided $i \neq 1$.

(iv) $(G, H, K)=\left(S L(V), S O(V), L_{i}\right)(p \neq 2)$. If $\operatorname{dim} V$ is even and $i>1$, the argument of (iii) shows $W \neq W_{H} W_{L}$, to complete the proof. We shall prove the result if $\operatorname{dim} V$ is even and $i=1$ in Lemma 4.8. So, we may assume $\operatorname{dim} V=2 n+1$ is odd and $1 \leq i \leq n$. Let $T_{H}<T$ be maximal tori of $H, G$ respectively, and $\varepsilon_{i}$ the usual weights of $T$. We can ensure that $\varepsilon_{i} \downarrow_{T_{H}}=-\varepsilon_{n+i} \downarrow_{T_{H}}=\mu_{i}$ for $1 \leq i \leq n$, and $\varepsilon_{2 n+1} \downarrow_{T_{H}}=0$. Then, $W_{L}$ is the stabilizer of $\omega_{i}=\varepsilon_{1}+\cdots+\varepsilon_{i}$, and $\left|W \cdot \omega_{i}\right|=\left(\begin{array}{c}2 n+1 \\ i\end{array}\right)$. 
Now, $W_{H}$ acts as all permutations and sign changes on $\pm \mu_{1}, \ldots, \pm \mu_{n}$. Hence, $\left|W_{H} \cdot \omega_{i}\right|=2^{i}\left(\begin{array}{c}n \\ i\end{array}\right)$. Hence, $W \neq W_{H} W_{L}$ for all $i$.

However, in all remaining cases in Table 4 (except $(G, H, K)=\left(S_{26}, F_{4}, N_{2}\right)$, when the condition of Lemma 4.5 fails), one can show that $W=W_{H} W_{L}$ is a factorization. So, Lemma 4.1 is of no use here, and we must treat them explicitly.

4.8. Lemma. There is no dense $H, K$-double coset in $G$ if

$$
(G, H, K)=\left(S L_{2 n}, S O_{2 n}, L_{1}\right)(p \neq 2) .
$$

Proof. Let $G=G L(V), H=S O(V)$, and let $v_{1}, \ldots, v_{2 n}$ be an orthonormal basis for $V$ with respect to the bilinear form $(\cdot, \cdot)$ preserved by $H$ on $V$. Let $f_{1}, \ldots, f_{2 n}$ be the corresponding dual basis for $V^{*}$. Then, the homomorphism defined by $\phi: f_{i} \mapsto v_{i}$ is $H$-equivariant. Let $K$ be the stabilizer of $v_{1} \otimes f_{1}$. Then, $G .\left(v_{1} \otimes f_{1}\right)=\{v \otimes f \in$ $\left.V \otimes V^{*} \mid f(v)=1\right\}$. Define $\theta: V \otimes V^{*} \rightarrow S^{2} V$ by $v \otimes f \mapsto v \cdot \phi(f)$, an $H$-equivariant morphism. Let $\bar{\theta}(g)=\theta\left(g .\left(v_{1} \otimes f_{1}\right)\right)$, so that $\bar{\theta}: G \rightarrow S^{2} V$ is constant on $H, K$ double cosets. Also, $\bar{\theta}(G)=\left\{v \cdot w \in S^{2} V \mid(v, w)=1\right\}$. Now, we can construct $S^{2} V$ as symmetric matrices, the matrix $M$ corresponding to the vector $\sum_{i, j} M_{i j} v_{i} \cdot v_{j}$. The action of $H$ on $S^{2} V$ is then just $x: M \mapsto x M x^{T}=x M x^{-1}$, as $x^{T}=x^{-1}$ for $x \in H$ when written as a matrix in terms of the orthonormal basis. So, the action is just conjugation. Now, one checks that as $\eta$ varies, the elements $\left(v_{1}+\eta v_{2}\right) \cdot v_{1} \in \bar{\theta}(G)$ give infinitely many non-conjugate semisimple matrices, and the proof is completed as usual by applying Lemma 1.4.

4.9. Lemma. Theorem $B$ holds if

$$
\begin{aligned}
& (G, H, K)=\left(S O_{2 n}, N_{2 i+1}, G L_{n}\right), \\
& (G, H, K)=\left(S p_{2 n}, N_{2 i}, G L_{n}\right)(p \neq 2) .
\end{aligned}
$$

Proof. If $(G, H, K)=\left(S O_{2 n}, G L_{n}, N_{1}\right)$, then $G=H K$ is a factorization by [LSS]. Excluding this case, we may assume that $i \geq 1$ and $p \neq 2$.

Let $e_{1}, \ldots, e_{n}, f_{1}, \ldots, f_{n}$ be a basis for the natural $G$-module $V$ with $\left(e_{i}, f_{j}\right)=$ $\delta_{i j},\left(e_{i}, e_{j}\right)=0=\left(f_{i}, f_{j}\right)$ for all $i, j$. Let $K$ be the connected stabilizer of the direct sum decomposition $V=\left\langle e_{1}, \ldots, e_{n}\right\rangle \oplus\left\langle f_{1}, \ldots, f_{n}\right\rangle$. Let $H$ be the connected stabilizer of the non-degenerate subspace $U=\left\langle e_{1}, \ldots, e_{i}, f_{1}, \ldots, f_{i}\right\rangle$ if $G=S p(V)$, or $U=\left\langle e_{1}, \ldots, e_{i}, f_{1}, \ldots, f_{i}, e_{n}+f_{n}\right\rangle$ if $G=S O(V)$. Let $L=V \otimes V$ and $z=$ $e_{1} \otimes f_{1}+\cdots+e_{n} \otimes f_{n}$; observe that in either case, $K$ fixes $z$. Moreover, $L \downarrow_{H} \cong$ $U \otimes U \oplus U \otimes U^{\perp} \oplus U^{\perp} \otimes U \oplus U^{\perp} \otimes U^{\perp}$. Let $\pi: L \rightarrow U \otimes U=M$ be projection along this direct sum decomposition, an $H$-equivariant morphism.

Now, the morphism $\theta: G \rightarrow M$ defined by $g \mapsto \pi(g z)$ sends $H, K$-double cosets in $G$ to $H$-orbits in $M$, so it suffices to show that there exist two closed $H$-orbits in $\pi(G z)$. Let $g_{\lambda} \in G$ be the map sending

$$
\begin{aligned}
& e_{1} \mapsto e_{1} \pm \lambda f_{i+1}, \quad e_{i+1} \mapsto \lambda f_{1}+e_{i+1}, \\
& f_{1} \mapsto e_{i+1}+(\lambda+1) f_{1}, \quad f_{i+1} \mapsto(\lambda+1) f_{i+1} \pm e_{1},
\end{aligned}
$$

where the ambiguous sign is chosen to be + if $G=S p(V),-$ if $G=S O(V)$. Now note that $\pi\left(g_{\lambda} z\right)=\pi(z)+\lambda\left(e_{1} \otimes f_{1} \pm f_{1} \otimes e_{1}\right)$. So as $\lambda$ varies, we obtain infinitely many elements of weight zero with respect to the maximal torus of $H$ which acts diagonally on the given basis. So Lemma 1.9 and (1.6) imply that there are infinitely many closed $H$-orbits in $\pi(G z)$, to complete the proof.

The next case will follow easily from the next general lemma. 
4.10. Lemma. Suppose $H, K, L<G$ are all connected algebraic groups. Suppose $H L$ and $K L$ are dense in $G$. Then, there is a dense $H \cap K, H \cap L$-double coset in $H$ if and only if there is a dense $H \cap K, K \cap L$-double coset in $K$.

Proof. Suppose $(H \cap K) x(H \cap L)$ is dense in $H$ for some $x \in H$. Then, $(H \cap K) x L$ is dense in $H L$ by Lemma 5.1, hence in $G$ as $H L$ is dense in $G$. So, it meets $K L$, as $G$ is an irreducible variety and $K L$ is dense in $G$. Therefore, it actually lies in $K L$, as $K L$ is stable under the action of $(H \cap K) \times L$ and $(H \cap K) x L$ is a single $(H \cap K) \times L$ orbit. So, $(H \cap K) x L=(H \cap K) y L$ for some $y \in K$, and this is dense in $K L$. Hence, by Lemma 5.1 again, $(H \cap K) y(K \cap L)$ is dense in $K$. The converse is the same.

4.11. Corollary. There is no dense $\bar{H}, \bar{K}$-double coset in $\bar{G}$ if

$$
(\bar{G}, \bar{H}, \bar{K})=\left(S O(V), N_{2}, B_{4}\right)
$$

where $V \downarrow_{K}=L_{K}\left(\omega_{4}\right)$.

Proof. Let $V$ be a spin module for $D_{5}$ and $B_{4} ; V$ possesses a non-degenerate $B_{4}$ invariant bilinear form. To apply Lemma 4.10, let $G=S L(V), H=D_{5}$, and $K=S O(V)$, so $H \cap K=B_{4}$, as $D_{5}$ does not preserve the bilinear form and $B_{4}$ is maximal in $D_{5}$. We show that there is a non-degenerate 2-space with $D_{5}$-stabilizer $A_{1} G_{2}$. Indeed, let $A_{1} G_{2}<S O_{3} \times S O_{7}<S O_{10}=D_{5}$ be the usual subgroup of $D_{5}$. Then, $V \downarrow_{A_{1} G_{2}}=L_{A_{1}}\left(\omega_{1}\right) \otimes \Delta_{7}$, where $\Delta_{7}$ is the 7-dimensional Weyl module for $G_{2}$. Now, $G_{2}$ fixes a 1 -space in $\Delta_{7}$; hence $A_{1} G_{2}$ fixes a 2 -space in $V$. But, the only connected subgroup of $D_{5}$ containing $A_{1} G_{2}$ is $N_{3}$, and $V \downarrow_{N_{3}}=L_{A_{1}}\left(\omega_{1}\right) \otimes L_{B_{3}}\left(\omega_{3}\right)$, so this fixes no 2 -space. Hence, there is some 2 -space $\omega$ in $\mathbb{P}^{2}(V)$ with $D_{5}$-stabilizer $A_{1} G_{2}$. Hence by dimension, $D_{5} . \omega$ is dense in $\mathbb{P}^{2}(V)$. But the non-degenerate 2spaces are also dense in $\mathbb{P}^{2}(V)$, and hence there is some non-degenerate 2-space $\omega_{1}$ in $D_{5} . \omega$; this will have $D_{5}$-stabilizer $A_{1} G_{2}$, as required.

Let $L=\operatorname{stab}_{G}\left(\omega_{1}\right)$, a parabolic subgroup of $G$. Then, $K \cap L$ is the group $N_{2}$ in $S O(V)$, and $H \cap L$ is $A_{1} G_{2}$. Hence, by dimensions, $H L$ and $K L$ are dense in $G$. Now, there is no dense $H \cap K, H \cap L$-double coset in $H$ (by Lemma 2.2 $H \cap K$ is $N_{1}$ in $H=S O_{10}$ and $H \cap L$ lies in $N_{3}$ ). Hence, by 4.10 there is no dense $H \cap K, K \cap L$-double coset in $K$, which is the required result.

It just remains to verify the last case

$$
(G, H, K)=\left(S O_{26-\delta_{p, 3}}, F_{4}, N_{2}\right)
$$

to complete the proof of Theorem B. This seems to be rather harder than the other cases, especially if $p=3$. For the remainder of the section, we work with the 27-dimensional module $V$ for $E=E_{6}$. The construction of $V$ described here is taken originally from Cohen and Cooperstein's paper [CC]. We shall use the notation defined in [B2, Section 4], and refer the reader to [B2] for fuller details.

Let $V$ be a 27-dimensional vector space over $k$ whose elements are triples $x=$ $\left[x_{1}, x_{2}, x_{3}\right]$ with $x_{i} \in M_{3}(k)$. We set

$$
\tilde{E}=\left\{g \in G L(V) \mid \text { there is } \lambda \in k^{\times} \text {such that, for all } x \in V, \mathcal{D}(g \cdot x)=\lambda \mathcal{D}(x)\right\},
$$

where $\mathcal{D}: V \rightarrow k$ is the cubic form $\mathcal{D}(x)=\operatorname{det} x_{1}+\operatorname{det} x_{2}+\operatorname{det} x_{3}-\operatorname{tr}\left(x_{1} x_{2} x_{3}\right)$. Then, $E=\tilde{E}^{\prime}$ is a simply connected simple algebraic group of type $E_{6}$, and $\tilde{E}$ is an extension of $E$ by a 1-dimensional torus. Let $e_{j k}^{i}$ be the element $\left[x_{1}, x_{2}, x_{3}\right]$ of $V$ all of whose entries are 0 , except the $j k$-entry of $x_{i}$, which is 1 . Let $e_{i}=e_{i i}^{1}$ for 
$i=1,2,3$, and $e=e_{1}+e_{2}+e_{3}$. Let $G=E_{e}$, a simple algebraic group of type $F_{4}$ (by $[\mathrm{CC}]$ ).

Note that $G$ preserves the non-degenerate symmetric bilinear form $(\cdot, \cdot)$ given by $(x, y)=\operatorname{tr}\left(x_{1} y_{1}+x_{2} y_{3}+x_{3} y_{2}\right)$ for $x=\left[x_{1}, x_{2}, x_{3}\right], y=\left[y_{1}, y_{2}, y_{3}\right] \in V$. Finally, the $G$-equivariant map \# : $V \rightarrow V, x \mapsto x^{\#}$ is defined by the identity

$$
\mathcal{D}(x+t y)=\mathcal{D}(x)+\left(x^{\#}, y\right) t+\left(x, y^{\#}\right) t^{2}+\mathcal{D}(y) t^{3},
$$

for $x, y \in V$ and $t$ an indeterminate. Explicitly, the map \# is given by

$$
x^{\#}=\left[x_{1}^{\#}-x_{2} x_{3}, x_{3}^{\#}-x_{1} x_{2}, x_{2}^{\#}-x_{3} x_{1}\right],
$$

where for $c \in M_{3}(k), c^{\#}$ is the adjoint of $c$ (the matrix whose $i j$-entry is the $j i$-cofactor of $c$ ).

We begin by defining a certain subgroup of $G$ of type $T_{1} G_{2} U_{14}$. Here, we exclude $p=2$, so that there are no degeneracies in the commutator relations. Let $P$ be the $B_{3}$-parabolic subgroup of $G$ obtained by deleting $\beta_{4}$ from the Dynkin diagram of $G$, labelling the simple roots of $G$ by $\beta_{1}, \ldots, \beta_{4}$ as in [B2, 4.6]. So $P=\left\langle B_{1}, K_{-\beta_{j}}\right| j=$ $1,2,3\rangle$. Let $P=L Q$, where $L=\left\langle T_{1}, K_{ \pm \beta_{j}} \mid j=1,2,3\right\rangle$ is a Levi factor and $Q$ is the unipotent radical. Let $L=L^{\prime} R$, where $R$ is the 1-dimensional radical. Observe that the subgroup $Z \cong G_{2}$ constructed in [B2, Lemma 4.10] is a subgroup of $L^{\prime}$.

By $[\mathrm{ABS}], Q$ has an $L$-composition series $Q=Q_{1}>Q_{2}>Q_{3}=0$ such that the factors $V_{i}=Q_{i} / Q_{i+1}$ are the $L^{\prime}$-modules $L_{B_{3}}\left(\omega_{3}\right)$ and $L_{B_{3}}\left(\omega_{1}\right)$ for $i=1,2$ respectively. As $L_{B_{3}}\left(\omega_{3}\right) \downarrow_{G_{2}}=L_{G_{2}}\left(\omega_{1}\right) \oplus k$, there is a unique $Z$-invariant subgroup $Q_{0} \triangleleft Q$ of dimension 14. We have thus defined a subgroup $H=R Z Q_{0}$ of type $T_{1} G_{2} U_{14}$. Let

$$
\begin{aligned}
\left(y_{\beta_{1}}(s), y_{\beta_{2}+2 \beta_{3}+\beta_{4}}(t)\right) & =y_{\beta_{1}+\beta_{2}+2 \beta_{3}+\beta_{4}}(A s t), \\
\left(y_{\beta_{3}}(s), y_{\beta_{1}+\beta_{2}+\beta_{3}+\beta_{4}}(t)\right) & =y_{\beta_{1}+\beta_{2}+2 \beta_{3}+\beta_{4}}(B s t),
\end{aligned}
$$

for some $A, B \in k^{\times}$, applying Chevalley's commutator formula. Let

$$
v(t)=y_{\beta_{2}+2 \beta_{3}+\beta_{4}}(B t) y_{\beta_{1}+\beta_{2}+\beta_{3}+\beta_{4}}(-A t) .
$$

Then a routine check using the commutator formula and the known action of root subgroups $K_{\beta_{i}}$ in [B2, 4.6] shows that each of the generators of $Z$ defined in [B2, Lemma 4.10] centralizes $V=\{v(t) \mid t \in k\}$. In particular, the image $V Q_{2} / Q_{2}$ in $V_{1}$ must be the 1 -space fixed by $Z$. So, $Q=Q_{0} V$.

4.12. Lemma. Let $H$ be the subgroup of $G=F_{4}$ of type $T_{1} G_{2} U_{14}$ defined above. Then, there is no dense $H, H$-double coset in $G$.

Proof. Recall that $H<P$, where $P$ is a $B_{3}$-parabolic subgroup. Let $n_{0}$ be a coset representative for the longest element of the Weyl group $N_{G}\left(T_{1}\right) / T_{1}$. Suppose $H g H$ is dense in $G$ for some $g \in G$; in particular, $\operatorname{dim} H \cap g H g^{-1}=6$. Now, $P n_{0} P$ is dense in $G$, so it must intersect $H g H$. Hence, as $H<P$, we have $H g H<P n_{0} P$, and $g=p_{1} n_{0} p_{2}$ for some $p_{1}, p_{2} \in P$. Hence, $\operatorname{dim}^{p_{1}} H \cap p_{2}^{-} H^{-}=6$, where $p_{2}^{-}={ }^{n_{0}} p_{2} \in P^{-}$, the opposite parabolic subgroup, and $H^{-}={ }^{n_{0}} H<P^{-}$. However, ${ }^{p_{1}} H \cap{ }^{p_{2}^{-}} H^{-}<$ $P \cap P^{-}=L$, so we consider ${ }^{p_{1}} H \cap L$. Write $p_{1}=l v x$ for $l \in L, x \in Q_{0}<H, v \in V$. Then, we showed above that $v$ centralizes $Z$, so ${ }^{p_{1}} H \cap L>{ }^{l} Z$. Arguing similarly for ${ }^{p_{2}^{-}} H^{-} \cap L$, we deduce that there are conjugates of $Z$ in $L$ with intersection of dimension $\leq 6$. But this implies that there is a dense $G_{2}, G_{2}$-double coset in $T_{1} B_{3}$, which is not the case by (1.8). 
We can now describe the orbits of $G$ on $e^{\perp} /\langle e\rangle$ when $p=3$, in terms of our basis $\left\{e_{j k}^{i} \mid 1 \leq i, j, k \leq 3\right\}$ for $V$.

4.13. Lemma. Suppose $p=3$ and $W=e^{\perp} /\langle e\rangle$ is the 25-dimensional module $L_{F_{4}}\left(\omega_{4}\right)$. Then, $G=F_{4}$ has 3 orbits on the 1-subspaces of $W$ with orbit representatives $l_{1}, l_{2}, l_{3}$, where $l_{i}=\left\langle u_{i}+\langle e\rangle\right\rangle$ and $u_{1}=e_{11}^{2}, u_{2}=e_{22}^{3}+e_{33}^{3}, u_{3}=e_{1}-e_{2}$. Moreover, $G_{l_{1}}=G_{\left\langle u_{1}\right\rangle}=P$, a $B_{3}$-parabolic subgroup, and $G_{l_{2}}=G_{\left\langle u_{2}\right\rangle}=H$ of type $T_{1} G_{2} U_{14}$.

Proof. We first prove the result on stabilizers. Observe that $l_{1}$ and $\left\langle u_{1}\right\rangle$ are spanned by highest weight vectors of weight $\omega_{4}$ in both $V, W$ by the computation of weights; hence both stabilizers equal $P=L Q$, a $B_{3}$-parabolic subgroup with Levi factor $L$ and unipotent radical $Q$. A direct calculation using the definition in [B2] shows that $\left\langle e, u_{2}\right\rangle^{\#} \subset\left\langle e, u_{1}, u_{2}\right\rangle$. Now, $G_{l_{2}}$ stabilizes $\left\langle e, u_{2}\right\rangle$, hence $\left\langle e, u_{2}\right\rangle^{\#}$, hence $\left\langle e, u_{1}, u_{2}\right\rangle$. But $\left\langle u_{1}\right\rangle$ is the only line $L$ in $\left\langle e, u_{1}, u_{2}\right\rangle$ with $L^{\#}=\{0\}$. As \# is $G$-equivariant, $G_{l_{2}}$ therefore stabilizes $\left\langle u_{1}\right\rangle$, so lies in $P$. Now, suppose $g \in G_{l_{2}}$. Then, $g \cdot u_{2}=\mu u_{2}+\nu e$ for some $\mu, \nu$. The weights of $e_{22}^{3}$ and $e_{33}^{3}$ are $\beta_{1}+\beta_{2}+\beta_{3}+\beta_{4}$ and $\beta_{2}+2 \beta_{3}+\beta_{4}$ in terms of the roots $\beta_{1}, \ldots, \beta_{4}$ of $G$, by restricting weights in [B2, Table 3]. In particular, they both involve $\beta_{4}$, whilst the weight of $e$ is 0 so does not. But then, $g \in P$ forces $\nu=0$. This shows that $G_{l_{2}}=G_{\left\langle u_{2}\right\rangle}$.

We now show that $H=R Z Q_{0}=G_{\left\langle u_{2}\right\rangle}$. For, $Z$ fixes $u_{2}$ by [B2, Lemma 4.10], and $R$ acts by scalars. A direct check shows that $K_{\beta_{4}}$ fixes $u_{2}$; this corresponds to the lowest weight vector of $L_{G_{2}}\left(\omega_{1}\right)=Q_{0} / Q_{2}$. Moreover, this extension is not split (it is not even abelian), so $K_{\beta_{4}}$ generates all of $Q_{0}$ as an $R Z$-group. So, $H<G_{\left\langle u_{2}\right\rangle}$. Conversely, take $l q \in G_{\left\langle u_{2}\right\rangle}$, where $l \in L, q \in Q$. If $l \notin R Z$, then $G_{\left\langle u_{2}\right\rangle}$ contains a conjugate of $L$, as $R Z Q / Q$ is maximal in $L Q / Q$. But this implies that the unipotent radical of $G_{\left\langle u_{2}\right\rangle}$, which contains $Q_{0}$, is all of $Q$. An explicit computation (using the commutator formula) shows that $K_{\beta_{1}+\beta_{2}+\beta_{3}+\beta_{4}}$ does not stabilize $\left\langle u_{2}\right\rangle$, so this is a contradiction. Hence, $l \in T_{1} Z$, so $q \in G_{\left\langle u_{2}\right\rangle}$, so $q \in Q_{0}$, so $l q \in T_{1} Z Q_{0}$. Hence, $H=G_{\left\langle u_{2}\right\rangle}$, as required.

It is now straightforward to deduce that there are just three orbits with representatives as given. Observe that $l_{1}, l_{2}$ are non-conjugate degenerate 1-spaces, whilst $l_{3}$ is non-degenerate. As $S O_{25}=F_{4} N_{1}(p=3)$ by [LSS], $G$ has just one orbit on non-degenerate lines, and $l_{3}$ is a representative. So, we need to show that there are precisely two orbits on degenerate lines. This is proved in $[\mathrm{CC}]$ over finite fields by a counting argument. Copying [B2, Lemma 4.7], it is easy to deduce from the finite fields case that $G$ can therefore have no more than two orbits on degenerate lines. But we have exhibited two disjoint orbits, and the result follows.

4.14. Proposition. There is no dense $G, K$-double coset in $L$ if

$$
(L, G, K)=\left(S O(W), F_{4}, N_{2}\right),
$$

where $W \downarrow_{G}=L_{G}\left(\omega_{4}\right)$.

Proof. Let $G$ be the group $F_{4}$ constructed above.

First, suppose $p \neq 3$. Then $W$ is just the module $e^{\perp}$, and $K$ is the stabilizer in $L$ of a vector $a \otimes b \in V \otimes V$, where $(a, a)=0=(b, b),(a, b)=1$ and $a, b \in e^{\perp}$. By [CC], we can define a $G$-equivariant map $*: V \otimes V \rightarrow V$ by $v_{1} \otimes v_{2} \mapsto\left(v_{1}+v_{2}\right)^{\#}-v_{1}^{\#}-v_{2}^{\#}$. It is sufficient to show that there are at least two disjoint closed $G$-orbits in $L .(a \otimes b)$. Consider the vector $\left(e_{22}^{2}+\eta e_{33}^{2}\right) \otimes\left((1-\eta) e_{22}^{3}+e_{33}^{3}\right) \in L .(a \otimes b)$. Its image under $*$ is $(\eta-1) e_{3}-\eta e_{2}$. This is of weight 0 relative to the maximal torus $T_{1}$ of $G$ (by 
[B2, Table 3]), so as $\eta$ varies we obtain infinitely many 0 -weight vectors. Hence, by Lemma 1.9 and (1.6) there are infinitely many closed $G$-orbits in $L .(a \otimes b)$, as required.

So, now suppose $p=3$. Let $H$ be the group of type $T_{1} G_{2} U_{14}$ constructed above. Let $W=e^{\perp} /\langle e\rangle$, which is isomorphic to $L_{G}\left(\omega_{4}\right)$. Choose $a, b \in W$ with $(a, a)=0=(b, b),(a, b)=1$. Suppose there is a dense $G, K$-double coset in $L$. As $K$ is the stabilizer of $a \otimes b \in W \otimes W$, this implies by dimension (conjugating if necessary) that $G_{a \otimes b}=G_{\langle a\rangle} \cap G_{\langle b\rangle}$ is of dimension 6 .

Suppose one of $a, b$ is $G$-conjugate to $u_{1}+\langle e\rangle$ as in Lemma 4.13; let it be $a$. Then, $G_{\langle a\rangle}=P$, a $B_{3}$-parabolic subgroup of dimension 37 , and $P$ lies in the parabolic subgroup $P_{1}$ of $L$ that stabilizes $\langle a\rangle$. Hence, the $P$-orbit of $\langle a\rangle$ is of dimension at most $\operatorname{dim} P_{1} \cdot\langle a\rangle=23$. So, $\operatorname{stab}_{P}(\langle b\rangle)$ is certainly of dimension at least $37-23>6$, a contradiction.

Hence, we may assume both $a$ and $b$ are $G$-conjugate to $u_{2}+\langle e\rangle$ by Lemma 4.13. But then, $G_{\langle a\rangle}$ and $G_{\langle b\rangle}$ are conjugate to the subgroup $H$ of type $T_{1} G_{2} U_{14}$ by Lemma 4.13, and $G_{\langle a\rangle} \cap G_{\langle b\rangle}$ of dimension 6 implies there is a dense $H, H$-double coset in $G$. This is not the case, by Lemma 4.12 .

This completes the proof of Theorem B.

\section{Proof of Theorem A}

We now deduce Theorem A from Theorem B and [B2]. Recall the notation $\mathcal{R}(G)$ from the introduction. Observe initially that if $H \in \mathcal{R}(G)$ and $K \in \mathcal{R}(H)$, then $K \in \mathcal{R}(G)$. Of course, in characteristic $0, \mathcal{R}(G)$ contains all reductive subgroups of $G$. In general, $\mathcal{R}(G)$ will contain very many but not necessarily all reductive subgroups of $G$. The main difficulty in deducing Theorem A is to show that $\mathcal{R}(G)$ is "closed under intersections" in the following sense: if $H, K \in \mathcal{R}(G)$ give rise to a factorization $G=H K$, then $H \cap K$ is in both $\mathcal{R}(H)$ and $\mathcal{R}(K)$. This need not be the case if $H K$ is not a factorization of $G$, even if the intersection is reductive.

We begin with an elementary lemma.

5.1. Lemma. Let $H, K \leq G$ and $Z \leq H$. If $x \in H$, then $Z x(H \cap K)$ is dense in $H$ if and only if $Z x K$ is dense in $H K$.

Proof. The bijective morphism $H / H \cap K \rightarrow H K / K$ defined by $x(H \cap K) \mapsto x K$ for $x \in H$ is a homeomorphism; for [H1, p. 56, ex. 4] implies that it is an open map. Hence, $Z x(H \cap K)$ is dense in $H$ if and only if $Z x K$ is dense in $H K$.

We say a semisimple group $G$ is of length $n$ if $G$ can be written as $G_{1} \cdot \ldots \cdot G_{n}$ is a commuting product of $n$ simple factors. If $G=G_{1} G_{2}$ is a commuting product of reductive (but not necessarily simple) factors, we say a subgroup $H<G$ is diagonally embedded if the projections $\pi_{i}: H \rightarrow G_{i}$ are bijective for each $i$. Note that if $H$ is diagonally embedded in $G$, then $H \in \mathcal{R}(G)$ - if the $G_{i}$ are simple then $H$ is maximal in $G$, and in the general case it is easy to write down a chain $H=H_{0}<H_{1}<\cdots<H_{n}=G$ with $H_{i}$ maximal in $H_{i+1}$ from this observation. We now consider diagonally embedded subgroups. The first lemma is well known.

5.2. Lemma ([B3, Lemma 4.4]). Let $G$ be a semisimple algebraic group of adjoint type of length at least 2. If $H \in \mathcal{M}(G)$, then one of the following holds:

(i) Some simple factor $1 \neq G_{1} \triangleleft G$ is contained in $H$.

(ii) $G$ is of length 2 and $H$ is diagonally embedded in $G$. 
We now consider double cosets of diagonally embedded subgroups as in Lemma 5.2(ii). We begin with a preliminary lemma.

5.3. Lemma. Let $G, G_{1}$ and $G_{2}$ be simple algebraic groups, where $G_{1}$ and $G_{2}$ are of adjoint type. Suppose that $\pi_{i}: G \rightarrow G_{i}$ is a bijective morphism (but not necessarily an isomorphism of algebraic groups) for $i=1,2$. Then, one of $\pi_{1} \circ \pi_{2}{ }^{-1}$ : $G_{2} \rightarrow G_{1}$ or $\pi_{2} \circ \pi_{1}^{-1}: G_{1} \rightarrow G_{2}$ is a morphism.

Proof. Let $(T, B ; \Sigma, \Pi)$ be a root system for $G$. For each $i$, let $B_{i}=\pi_{i}(B), T_{i}=$ $\pi_{i}(T)$ for each $i$, hence defining a root system $\left(T_{i}, B_{i} ; \Sigma_{i}, \Pi_{i}\right)$ for $G_{i}$. Let $E=$ $X(T) \otimes_{\mathbb{Z}} \mathbb{R}$ and $E_{i}=X\left(T_{i}\right) \otimes_{\mathbb{Z}} \mathbb{R}$, and fix inner products on $E$ and $E_{i}$ invariant under the corresponding Weyl group. Given non-zero vectors $v$ and $w$ in either of these spaces, let $R(v, w)$ denote the ratio of their lengths.

Denote the root subgroups of $G$ and $G_{i}$ by $U_{\alpha}(\alpha \in \Sigma)$ and $U_{i, \alpha}\left(\alpha \in \Sigma_{i}\right)$ respectively. The map $\pi_{i}: G \rightarrow G_{i}$ sends the set of root subgroups of $G$ bijectively onto the root subgroups of $G_{i}$, hence induces a bijection $\theta_{i}: \Sigma \rightarrow \Sigma_{i}$ such that $\pi_{i}\left(U_{\alpha}\right)=U_{i, \theta_{i}(\alpha)}$ for all $\alpha \in \Sigma$. Let $\bar{\pi}_{i}: T \rightarrow T_{i}$ denote the restriction of $\pi_{i}$ to $T$, and let $\pi_{i}^{*}: X\left(T_{i}\right) \rightarrow X(T)$ denote the associated comorphism. Since $\bar{\pi}_{i}$ is surjective, $\pi_{i}^{*}$ is injective. Fix parametrizations $x_{\alpha}: k \rightarrow U_{\alpha}(\alpha \in \Sigma)$ and $x_{i, \alpha}: k \rightarrow U_{i, \alpha}\left(\alpha \in \Sigma_{i}\right)$ of the root subgroups. As $\pi_{i}$ is a morphism, $\pi_{i}\left(x_{\alpha}(t)\right)=x_{i, \theta_{i}(\alpha)}\left(c_{i, \alpha} t^{q_{i, \alpha}}\right)$ for coefficients $c_{i, \alpha} \in k^{\times}$and certain powers $q_{i, \alpha}$ of $p$, depending on $\alpha \in \Sigma$ [if $p=0$, $q_{i, \alpha}=1$ always].

We claim that either $q_{1, \alpha} \geq q_{2, \alpha}$ for all $\alpha \in \Sigma$, or $q_{1, \alpha} \leq q_{2, \alpha}$ for all $\alpha \in \Sigma$. Well, otherwise, $p \neq 0$ and we can find $\alpha, \beta \in \Sigma$ such that $q_{1, \alpha}>q_{2, \alpha}$ and $q_{1, \beta}<q_{2, \beta}$. A simple calculation shows that $\pi_{i}^{*}\left(\theta_{i}(\alpha)\right)=q_{i, \alpha} \alpha$ for all $\alpha \in \Sigma$, so $R\left(\theta_{i}(\alpha), \theta_{i}(\beta)\right)=$ $R\left(q_{i, \alpha} \alpha, q_{i, \beta} \beta\right)=\frac{q_{i, \alpha}}{q_{i, \beta}} R(\alpha, \beta)$ for each $i$. So,

$$
\frac{R}{p} \geq \frac{q_{1, \beta}}{q_{2, \beta}} R\left(\theta_{1}(\alpha), \theta_{1}(\beta)\right)=\frac{q_{1, \alpha}}{q_{2, \alpha}} R\left(\theta_{2}(\alpha), \theta_{2}(\beta)\right) \geq \frac{p}{R},
$$

where $R$ is the ratio of a long root to a short root in $\Sigma$. This implies that $R^{2} \geq$ $p^{2} \geq 4$ which is a contradiction as $R^{2}$ equals 1,2 or 3 .

So, without loss of generality, assume that $q_{1, \alpha} \geq q_{2, \alpha}$ for all $\alpha \in \Sigma$. We claim in this case that $\pi_{1} \circ \pi_{2}^{-1}$ is a morphism. Arguing as in [LS, Lemma 1.2], it suffices to check that the restriction of $\pi_{1} \circ \pi_{2}^{-1}$ to each root subgroup and to $T_{2}$ is a morphism. On the root subgroup $U_{2, \theta_{2}(\alpha)}$ for $\alpha \in \Sigma, \pi_{1} \circ \pi_{2}^{-1}$ is the map $x_{2, \theta_{2}(\alpha)}(t) \mapsto x_{1, \theta_{1}(\alpha)}\left(c_{\alpha} t^{q_{\alpha}}\right)$, where $c_{\alpha}=c_{1, \alpha} / c_{2, \alpha}$ and $q_{\alpha}=q_{1, \alpha} / q_{2, \alpha}$, which is a morphism as $q_{1, \alpha} \geq q_{2, \alpha}$ and both are powers of $p$ [or 1 if $p=0$ ]. It remains to check that the restriction of $\pi_{1} \circ \pi_{2}^{-1}$ to $T_{2}$ is a morphism. Now, $q_{1, \alpha}$ is an integer multiple of $q_{2, \alpha}$. So, $\pi_{1}^{*}\left(\Sigma_{1}\right)$ is contained in $\pi_{2}^{*}\left(\Sigma_{2}\right)$. As $G_{1}$ is of adjoint type, $X\left(T_{1}\right)$ is generated as an abelian group by $\Sigma_{1}$, so in fact $\pi_{1}^{*}\left(X\left(T_{1}\right)\right)$ is a subgroup of $\pi_{2}^{*}\left(X\left(T_{2}\right)\right)$. Hence, there is a well-defined homomorphism of abelian groups $\left(\pi_{2}^{*}\right)^{-1} \circ \pi_{1}^{*}: X\left(T_{1}\right) \rightarrow X\left(T_{2}\right)$. This induces a morphism $T_{2} \rightarrow T_{1}$ of tori, which necessarily equals the restriction of $\pi_{1} \circ \pi_{2}^{-1}$ to $T_{2}$. Consequently, this restriction is a morphism, completing the proof.

5.4. Proposition. Suppose $G=G_{1} G_{2}$ is a semisimple, adjoint algebraic group of length 2. If $H, K<G$ are diagonally embedded subgroups, then either $G=H K$ or there is no dense $H, K$-double coset in $G$.

Proof. We may conjugate to assume that the double coset $H K$ is closed. Let $\pi_{i}: H \rightarrow G_{i}$ and $\delta_{i}: K \rightarrow G_{i}$ be the projections, bijective morphisms for each $i$. 
By Lemma 5.3, one of $\pi_{1} \circ \pi_{2}^{-1}$ or $\pi_{2} \circ \pi_{1}^{-1}$ is a morphism; assume without loss of generality that $\pi_{2} \circ \pi_{1}^{-1}$ is a morphism. Then, either $\delta_{1} \circ \delta_{2}^{-1}$ is a morphism, in which case $\delta_{1} \circ \delta_{2}^{-1} \circ \pi_{2} \circ \pi_{1}^{-1}$ is a morphism, or both $\pi_{2} \circ \pi_{1}^{-1}$ and $\delta_{2} \circ \delta_{1}^{-1}$ are bijective morphisms from $G_{1}$ to $G_{2}$. In the latter case, Lemma 5.3 again implies that either $\pi_{2} \circ \pi_{1}^{-1} \circ \delta_{1} \circ \delta_{2}^{-1}$ or $\delta_{2} \circ \delta_{1}^{-1} \circ \pi_{1} \circ \pi_{2}^{-1}$ is a morphism. We have shown that at least one of the maps $\pi_{1} \circ \pi_{2}^{-1} \circ \delta_{2} \circ \delta_{1}^{-1}, \pi_{2} \circ \pi_{1}^{-1} \circ \delta_{1} \circ \delta_{2}^{-1}, \delta_{1} \circ \delta_{2}^{-1} \circ \pi_{2} \circ \pi_{1}^{-1}$ or $\delta_{2} \circ \delta_{1}^{-1} \circ \pi_{1} \circ \pi_{2}^{-1}$ is a morphism.

Now assume without loss of generality that $\pi_{1} \circ \pi_{2}^{-1} \circ \delta_{2} \circ \delta_{1}^{-1}$ is a morphism, the other three cases being entirely similar. Let $\theta=\pi_{2} \circ \pi_{1}^{-1}: G_{1} \rightarrow G_{2}$ and $\phi=\delta_{2} \circ$ $\delta_{1}^{-1}: G_{1} \rightarrow G_{2}$, both isomorphisms of abstract groups. Then, $H=\left\{g \theta(g) \mid g \in G_{1}\right\}$ and $K=\left\{g \phi(g) \mid g \in G_{1}\right\}$. Hence, $H \cap K=\left\{g \theta(g) \mid g \in G_{1}, \theta(g)=\phi(g)\right\} \cong G_{1}^{\sigma}$, where $\sigma=\theta^{-1} \circ \phi$ is an abstract automorphism of $G_{1}$ which by assumption is a morphism of algebraic groups. So now we can apply [S1, 10.13] to deduce that there are two distinct possibilities:

(i) $G_{1}^{\sigma}$ is finite. Then, $\operatorname{dim} H K=\operatorname{dim} H+\operatorname{dim} K-\operatorname{dim} H \cap K=\operatorname{dim} G$. Thus $H K$ is dense and closed, so $G=H K$ is a factorization.

(ii) $\sigma$ is an algebraic automorphism of $G_{1}$. Consider an arbitrary double coset $H h K$ with $h \in G_{1}$, with stabilizer $h^{-1} H h \cap K=\left\{g \theta(g) \mid g \in G_{1}, \theta\left(h g h^{-1}\right)=\phi(g)\right\}$. Let $\operatorname{Int}(h)$ be the inner automorphism of $G_{1}$ defined by $h \in G_{1}$. Then, $h^{-1} H h \cap K$ is isomorphic to the fixed points of $\operatorname{Int}(h)^{-1} \circ \sigma$ in $G_{1}$. But this is an algebraic automorphism of $G_{1}$, so again by [S1, 10.13], the set of fixed points $h^{-1} H h \cap K$ is of positive dimension. Hence, $H h K$ is not dense in $G$, for all $h \in G_{1}$.

5.5. Remark. This proof gives rise to some interesting 'diagonal' factorizations. For example, let $G=G_{1} G_{2}$ be a product of two isomorphic simple factors, where $\theta: G_{1} \rightarrow G_{2}$ is an isomorphism. Let $\sigma: G_{1} \rightarrow G_{1}$ be a Frobenius automorphism of $G_{1}(p \neq 0)$. Then, $G=H K$ is a factorization if $H=\left\{g \theta(g) \mid g \in G_{1}\right\}$ and $K=\left\{g \theta(\sigma(g)) \mid g \in G_{1}\right\}$. The existence of these factorizations can also be proved using Lang's theorem.

5.6. Lemma. Let $G$ be simple, and suppose $G=H K$, where $H, K \in \mathcal{M}(G)$. Then $H \cap K$ is in both $\mathcal{R}(H)$ and $\mathcal{R}(K)$.

Proof. We verify this explicitly for each entry in Table 1. First, we claim that if $H<G$ is a connected reductive subgroup normalized by some maximal torus $T$ of $G$, then $H \in \mathcal{R}(G)$. Indeed, clearly $H \in \mathcal{R}(H T)$, so we may assume $T<$ $H$. Moreover, by induction, we may assume $H$ is a maximal connected reductive subgroup. Then, either $H$ has some central torus, so that $H$ is a Levi factor, or $H$ is a maximal connected subgroup. In either case $H \in \mathcal{M}(G)$, proving the claim. In particular, this observation proves the lemma if $G$ is exceptional, when the possible factorizations are determined in [B2], or if $(G, H, K, p)=\left(S p_{2 n}, N_{i}, S O_{2 n}, 2\right)$, since in either case both $H$ and $K$ are maximal rank, so $H \cap K$ is of maximal rank in both $H$ and $K$. We now consider the remaining cases in Table 1; it is sufficient to do this up to graph automorphisms of $G$.

(i) We first consider the first three entries in Table 1. Here the intersections are given in the table below:

\begin{tabular}{llll}
$G$ & $H$ & $K$ & $(H \cap K)^{0}$ \\
\hline$S L_{2 n}$ & $S p_{2 n}$ & $G L_{2 n-1}$ & $T_{1} S p_{2 n-2}$ \\
$S O_{2 n}$ & $S O_{2 n-1}$ & $G L_{n}$ & $G L_{n-1}$ \\
$S O_{4 n}$ & $S O_{4 n-1}$ & $S p_{2} \otimes S p_{2 n}$ & $S p_{2} \times S p_{2 n-2}$
\end{tabular}


In the first two cases, $H \cap K$ and its embedding in $H$ and $K$ are straightforward to compute and the result follows. In the third case, we can find a subgroup $Z \cong S p_{2} \times S p_{2 n-2}$ of $G$ such that, if $V$ is the natural module for $G$,

$$
\begin{aligned}
V \downarrow_{Z} & \cong L_{Z}\left(\omega_{1}, 0\right) \otimes\left(L_{Z}\left(\omega_{1}, 0\right) \oplus L_{Z}\left(0, \omega_{1}\right)\right) \\
& \cong\left(L_{Z}\left(\omega_{1}, 0\right) \otimes L_{Z}\left(\omega_{1}, 0\right)\right) \oplus\left(L_{Z}\left(\omega_{1}, 0\right) \otimes L_{Z}\left(0, \omega_{1}\right)\right) .
\end{aligned}
$$

From the first isomorphism here, we see that $Z$ is a subgroup of $K=S p_{2} \otimes S p_{2 n}$, and there is a chain of embeddings $S p_{2} \times S p_{2 n-2}<S p_{2} \otimes\left(S p_{2} S p_{2 n-2}\right)<S p_{2} \otimes S p_{n}$, each maximal in the next, proving that $Z \in \mathcal{R}(K)$. On the other hand, from the second isomorphism, we see that $Z<S_{4} S_{4 n-4}$. Now, the diagonal subgroup $S p_{2}<S p_{2} \otimes S p_{2}<S O_{4}$ fixes a non-singular line in the 4-dimensional orthogonal space (if $e, f$ is a symplectic basis then $S p_{2}$ fixes $e \otimes f-f \otimes e$ ), and this identifies $S p_{2}$ with the subgroup $S O_{3}<S O_{4}$. Hence, we have a chain of embeddings $S p_{2} \times$ $S p_{2 n-2}<S p_{2}\left(S p_{2} \otimes S p_{2 n-2}\right)=S O_{3}\left(S p_{2} \otimes S p_{2 n-2}\right)<S O_{3} S O_{4 n-4}<S O_{4 n-1}$ proving that $Z \in \mathcal{R}(H)$. Finally, dimension implies $Z$ is indeed equal to $(H \cap K)^{0}$.

(ii) We next consider the factorizations involving $G_{2}$ or $B_{3}$ in Table 1 . By applying graph automorphisms to $G=\mathrm{PSO}_{8}$, it is sufficient to prove the lemma for the cases in the table below - we have already treated the cases $(G, H, K)=$ $\left(S O_{8}, N_{1}, G L_{4}\right)$ and $\left(S O_{8}, N_{1}, S p_{2} \otimes S p_{4}\right)$ in (i):

\begin{tabular}{lllll}
$G$ & $p$ & $H$ & $K$ & $(H \cap K)^{0}$ \\
\hline$S p_{6}$ & $p=2$ & $S O_{6}$ & $G_{2}$ & $A_{2}$ \\
$S O_{7}$ & $p \neq 2$ & $N_{1}$ & $G_{2}$ & $A_{2}$ \\
$S p_{6}$ & $p=2$ & $N_{2}$ & $G_{2}$ & $A_{1} \tilde{A}_{1}$ \\
$S O_{7}$ & $p \neq 2$ & $N_{2}$ & $G_{2}$ & $A_{1} T_{1}$ \\
$S O_{8}$ & & $N_{1}$ & $B_{3}$ or ${ }^{\tau} B_{3}$ & $G_{2}$
\end{tabular}

In the first two cases, the intersection must have dimension 8 , so the only possibility is the long root subgroup $A_{2}$ of $G_{2}$ (or possibly $\tilde{A}_{2}$ if $p=3$ ); this is of maximal rank in $G_{2}$ so certainly lies in $\mathcal{R}(K)$. The embedding $A_{2}<S O_{6}$ is well known; it is $A_{2}<G L_{3}<S O_{6}$, so $H \cap K \in \mathcal{R}(H)$. In the final case, $G_{2}$ fixes a non-singular 1-space in $L_{B_{3}}\left(\omega_{3}\right)$, so $N_{1} \cap B_{3}$ contains $G_{2}$, hence equals $G_{2}$ by dimension. Here, $H \cap K$ is maximal in both $H$ and $K$, so the result follows.

To consider the third and fourth cases, let $V$ be the natural module for $G$ and compute the restriction $V \downarrow_{A_{1} \tilde{A}_{1}}$, where $A_{1} \tilde{A}_{1}$ is the maximal subgroup of $G_{2}$. By considering weights, the restriction splits as $L_{\tilde{A}_{1}}\left(2 \omega_{1}\right) \oplus\left(L_{A_{1}}\left(\omega_{1}\right) \otimes L_{\tilde{A}_{1}}\left(\omega_{1}\right)\right)$. Hence, if $p=2, A_{1} \tilde{A}_{1} \leq N_{2} \cap G_{2}$, hence equals the intersection by dimension. This is of maximal rank in $G_{2}$, so we just need to show that $H \cap K \in \mathcal{R}(H)$. For this, there is a chain of subgroups $A_{1} \tilde{A}_{1}<S p_{2} S O_{4}<S p_{2} S p_{4}=N_{2}$ with each maximal in the next, proving the result. Finally, if $p \neq 2$ then the subgroup $A_{1} T_{1}<A_{1} \tilde{A}_{1}$ is of the correct dimension to be the intersection $(H \cap K)^{0}$, and is a maximal rank subgroup of $G_{2}$. So we just need to show that $A_{1} T_{1} \in \mathcal{R}(H)$. Here it is clear from the structure of $V \downarrow_{A_{1} \tilde{A}_{1}}$ that $A_{1} T_{1}<G L_{2} T_{1}<S O_{4} T_{1}<S O_{5} T_{1}=N_{2}$ is a chain of embeddings proving $A_{1} T_{1} \in \mathcal{R}(H)$. 
(iii) For the remaining cases, the intersection is computed in [LSS, Proposition $1.9]$ :

\begin{tabular}{lllll}
$G$ & $p$ & $H$ & $K$ & $(H \cap K)^{0}$ \\
\hline$S O_{56}$ & $p=2$ & $E_{7}$ & $N_{1}$ & $E_{6}$ \\
$S O_{32}$ & $p=2$ & $D_{6}$ & $N_{1}$ & $A_{5}$ \\
$S O_{25}$ & $p=3$ & $F_{4}$ & $N_{1}$ & $D_{4}$ \\
$S O_{20}$ & $p=2$ & $A_{5}$ & $N_{1}$ & $A_{2} A_{2}$ \\
$S O_{16}$ & & $B_{4}$ & $N_{1}$ & $B_{3}$ \\
$S O_{13}$ & $p=3$ & $C_{3}$ & $N_{1}$ & $A_{1} A_{1} A_{1}$
\end{tabular}

In each case, it is shown in [LSS] that $(H \cap K)^{0}$ is normalized by some maximal torus of $H$, so it just remains to show that $H \cap K \in \mathcal{R}(K)$. For this, we just exhibit a chain of subgroups proving that $H \cap K \in \mathcal{R}(K)$, leaving the details to the reader; for this, the embeddings are explained in more detail in [LSS, Proposition 1.9].

$$
\begin{gathered}
E_{6} \stackrel{\omega_{1}}{<} S L_{27}<G L_{27}<S O_{54}<S O_{55}=N_{1}, \\
A_{5} \stackrel{\omega_{2}}{<} S L_{15}<G L_{15}<S O_{30}<S O_{31}=N_{1}, \\
D_{4} \stackrel{\omega_{1}, \omega_{3}, \omega_{4}}{<} S O_{8} S O_{8} S O_{8}<S O_{8} S O_{16}<S O_{24}=N_{1}, \\
A_{2} A_{2} \stackrel{\omega_{1} \otimes \omega_{2}}{<} S L_{9}<G L_{9}<S O_{18}=N_{1}, \\
B_{3} \stackrel{\omega_{1}, \omega_{3}}{<} S O_{7} S O_{7}<S O_{7} S O_{8}<S O_{15}=N_{1}, \\
A_{1} A_{1} A_{1}<S O_{4} S O_{4} S O_{4}<S O_{4} S O_{8}<S O_{12}=N_{1} .
\end{gathered}
$$

In the last case here, the embedding $A_{1} A_{1} A_{1}<S O_{4} S O_{4} S O_{4}$ is such that the highest weights of $A_{1} A_{1} A_{1}$ on the $S O_{4}$ factors are $\omega_{1} \otimes \omega_{1} \otimes 0, \omega_{1} \otimes 0 \otimes \omega_{1}, 0 \otimes \omega_{1} \otimes \omega_{1}$ respectively.

5.7. Lemma. Suppose $G$ is a connected reductive algebraic group and $H, K \in$ $\mathcal{M}(G)$ are such that $G=H K$ is a factorization. Then, $H \cap K$ is in both $\mathcal{R}(H)$ and $\mathcal{R}(K)$.

Proof. We prove that $H \cap K \in \mathcal{R}(K)$ by induction on $\operatorname{dim} G$, the case $G=1$ being trivial. First suppose $G$ is semisimple. If $G$ is of length 1, then the result is precisely Lemma 5.6. So, $G$ is of length greater than 1 . Also, we may replace $G$ by the corresponding adjoint group $\operatorname{Ad} G$, as $\operatorname{Ad} \mathcal{R}(K)=\mathcal{R}(\operatorname{Ad} K)$. So by Lemma 5.2 there are two cases:

(i) Some simple factor $1 \neq G_{1} \triangleleft G$ is contained in $H$. Then, $H / G_{1} \in \mathcal{M}\left(G / G_{1}\right)$. Notice that either $K G_{1} / G_{1}=G / G_{1}$ (which will not cause problems) or $K G_{1} / G_{1} \in$ $\mathcal{M}\left(G / G_{1}\right)$. By induction, $\left(H / G_{1}\right) \cap\left(K G_{1} / G_{1}\right) \in \mathcal{R}\left(K G_{1} / G_{1}\right)$. Now, $H \cap\left(K G_{1}\right)=$ $(H \cap K) G_{1}$. Let $A=H \cap K$ and $B=A G_{1} / G_{1}$. Then as $B \in \mathcal{R}\left(K G_{1} / G_{1}\right)$, there is a chain of subgroups $B^{0}=B_{0}<B_{1}<\cdots<B_{n}=K G_{1} / G_{1}$ with $B_{i} \in \mathcal{M}\left(B_{i+1}\right)$ for each $i$. Let $A_{i}$ be the connected pre-image of $B_{i}$ under $q: K \rightarrow K G_{1} / G_{1}$. Then, $A_{0}=\left(\left(A G_{1}\right) \cap K\right)^{0}=\left(H \cap\left(K G_{1}\right) \cap K\right)^{0}=(H \cap K)^{0}=A$. So, we obtain a chain of subgroups $A=A_{0}<A_{1}<\cdots<A_{n}=K$ with each $A_{i} \in \mathcal{M}\left(A_{i+1}\right)$. So, $H \cap K \in \mathcal{R}(K)$, as required.

(ii) $G$ is of length 2 and $H$ is diagonally embedded in $G$. Again by Lemma 5.2 there are two cases for $K$. First, suppose $K$ is also diagonally embedded. Then, $\operatorname{dim} H \cap K=\operatorname{dim} H+\operatorname{dim} K-\operatorname{dim} G=0$, so $H \cap K$ is finite and the result follows. Otherwise, some simple factor of $G$ is contained in $K$. Without loss of generality, 
suppose $G=G_{1} G_{2}$ with each $G_{i}$, simple and that $G_{2} \leq K$. Then, $K=K_{1} G_{2}$ with $K_{1} \in \mathcal{M}\left(G_{1}\right)$. Let $\pi_{i}: H \rightarrow G_{i}$ be the projections, bijective morphisms. Then, $H \cap K=\pi_{1}^{-1} K_{1}$. Let $K_{2}=\left\{\pi_{1}\left(x_{1}\right) \pi_{2}\left(x_{2}\right) \mid x_{1}, x_{2} \in H \cap K\right\}$. Then, $H \cap K$ is diagonally embedded in $K_{2}$, so that $H \cap K \in \mathcal{R}\left(K_{2}\right)$, and $K_{2} \in \mathcal{M}(K)$. Hence, $H \cap K \in \mathcal{R}(K)$.

Finally, suppose $G$ is reductive and not semisimple. Let $R \neq 1$ be the radical of $G$. If $H R=G$ then $G^{\prime} \leq H$, so $K^{\prime} \leq H \cap K \leq K$, and it is clear from this that $H \cap K \in \mathcal{R}(K)$. So, we may assume $H R \neq G$, so that by maximality, $H R=H$. Then the argument of (i) (with $G_{1}=R$ ) gives the result.

We are finally in a position to prove that $\mathcal{R}(G)$ is closed under intersections in full generality.

5.8. Proposition. Suppose $G$ is a reductive algebraic group and $H, K \in \mathcal{R}(G)$ are such that $G=H K$ is a factorization. Then, $H \cap K$ is in both $\mathcal{R}(H)$ and $\mathcal{R}(K)$.

Proof. Again we use induction on $\operatorname{dim} G$, the case $G$ finite being trivial. We may assume $G, H, K$ are connected, so let $H, K \in \mathcal{R}(G)$ be connected. We may embed $H \leq H_{1}, K \leq K_{1}$ with $H_{1}, K_{1} \in \mathcal{M}(G)$ and $H \in \mathcal{R}\left(H_{1}\right), K \in \mathcal{R}\left(K_{1}\right)$. Then, $G=H K$ implies

$$
H_{1}=H\left(H_{1} \cap K\right), \quad K_{1}=\left(H \cap K_{1}\right) K .
$$

In particular, $H_{1}=H\left(H_{1} \cap K_{1}\right)$. By Lemma 5.7, $H_{1} \cap K_{1} \in \mathcal{R}\left(H_{1}\right)$, and $H \in \mathcal{R}\left(H_{1}\right)$ by definition, so induction implies that $H \cap K_{1}$ is in both $\mathcal{R}(H)$ and $\mathcal{R}\left(H_{1} \cap K_{1}\right)$. Similarly, $H_{1} \cap K$ is in both $\mathcal{R}(K)$ and $\mathcal{R}\left(H_{1} \cap K_{1}\right)$.

Now, $H_{1}=H\left(H_{1} \cap K\right)$ implies $H_{1} \cap K_{1}=\left(H \cap K_{1}\right)\left(H_{1} \cap K\right)$. We have shown that $H \cap K_{1}$ and $H_{1} \cap K$ are in $\mathcal{R}\left(H_{1} \cap K_{1}\right)$. Hence, by induction, $H \cap K$ is in both $\mathcal{R}\left(H \cap K_{1}\right)$ and $\mathcal{R}\left(H_{1} \cap K\right)$. The result follows, as $H \cap K_{1}$ is in $\mathcal{R}(H)$ and $H_{1} \cap K$ is in $\mathcal{R}(K)$.

Now we can prove Theorem A. For convenience, we restate the theorem:

5.9. Theorem. Let $G$ be a connected reductive algebraic group, and take $H, K \in$ $\mathcal{R}(G)$. Then, either $G=H K$ or there is no dense $H, K$-double coset in $G$.

Proof. We prove this by induction on $\operatorname{dim} G$; the induction starts with $G=1$. So, let $G$ be a connected reductive group of dimension $d$ and suppose the result holds for all groups of dimension less than $d$.

(i) We first show that the result holds if $H, K \in \mathcal{M}(G)$ and $G$ is semisimple. If $G$ is of length 1, then the result holds by Theorem B, or by [B2] if $G$ is exceptional. So suppose $G$ is of length greater than 1. By Lemma 1.2, we may assume $G$ is adjoint, so Lemma 5.2 applies. Suppose first that some simple factor $1 \neq G_{1}$ of $G$ is contained in $H$. Then, if $G_{1} K=G, G=H K$, and the result follows. Otherwise, maximality implies $G_{1} K=K$ and the result follows by induction from the case $\left(G / G_{1}, H / G_{1}, K / G_{1}\right)$. A similar argument applies if some simple factor of $G$ is contained in $K$. Hence, both $H$ and $K$ are diagonally embedded and $G$ is of length 2. Now the result follows by Proposition 5.4.

(ii) We now show that the result holds if $H, K \in \mathcal{M}(G)$ and $G$ is reductive. Conjugating, we may assume that $H K$ is closed. Let $R$ be the radical of $G$. Then, if $H K R=G$, either $H K=G$, as required, or we can find $r \in R-H K$. In this case, $H r K=H K r$ is also closed and disjoint from $H K$. Hence, there are at least two disjoint closed $H, K$-double cosets in $G$, and the result follows by Lemma 1.4. So, we 
may assume $H K R \neq G$. Then, $H R \neq G$ and $K R \neq G$, so by maximality, $H R=H$, $K R=K$. Then, the result follows by induction from the case $(G / R, H / R, K / R)$.

(iii) We now show that the result holds if $H, K \in \mathcal{R}(G)$ and $G$ is reductive. Observe that, by Lemma 1.2, we may assume $H, K$ are connected. So, let $H, K \in$ $\mathcal{R}(G)$ be connected. Then, we may embed $H \leq H_{1}, K \leq K_{1}$ with $H_{1}, K_{1} \in \mathcal{M}(G)$ such that $H \in \mathcal{R}\left(H_{1}\right), K \in \mathcal{R}\left(K_{1}\right)$. If $G \neq H_{1} K_{1}$, then there is no dense $H_{1}, K_{1}$ double coset in $G$ by (ii), so the result holds. So, suppose $G=H_{1} K_{1}$; if $H=$ $H_{1}, K=K_{1}$, then $G=H K$ and we are done. So, assume without loss of generality that $H \neq H_{1}$. Since $G=H_{1} K_{1}$, Proposition 5.8 implies $H_{1} \cap K_{1} \in \mathcal{R}\left(H_{1}\right)$. So by induction either $H_{1}=H\left(H_{1} \cap K_{1}\right)$ or there is no dense $H, H_{1} \cap K_{1}$-double coset in $H_{1}$. In the latter case Lemma 5.1 implies there is no dense $H, K_{1}$-double coset in $H_{1} K_{1}=G$, and the result follows. In the former case, $G=H_{1} K_{1}=H K_{1}$. Hence, by Proposition 5.8, $H \cap K_{1} \in \mathcal{R}\left(K_{1}\right)$, and also $K \in \mathcal{R}\left(K_{1}\right)$ by definition. So by induction, either $K_{1}=\left(H \cap K_{1}\right) K$ or there is no dense $H \cap K_{1}, K$-double coset in $K_{1}$. But the former case implies $G=H K_{1}=H K$, and in the latter case there is no dense $H, K$-double coset in $H K_{1}=G$ by Lemma 5.1.

This completes the proof of the main results of the paper.

\section{ACKNOWLEDGEMENTS}

I would like to thank my Ph.D. supervisor, Professor Martin Liebeck, for all his support, and the referee for correcting the proof of Proposition 5.4. This work was supported by the EPSRC.

\section{REFERENCES}

[ABS] H. Azad, M. Barry, and G. M. Seitz, On the structure of parabolic subgroups, Comm. in Alg., 18:551-562, 1990. MR 91d:20048

[Borel] A. Borel, Linear Algebraic Groups, Springer-Verlag, New York, second edition, 1991. MR 92d:20001

[B1] J. W. Brundan, Double cosets in algebraic groups, PhD thesis, Imperial College, London, 1996.

[B2] L Double coset density in exceptional algebraic groups, to appear in J. London Math. Soc., 58:63-83, 1998. CMP 99:07

[B3] _ Multiplicity-free subgroups of reductive algebraic groups, J. Algebra, 188:310330, 1997. MR 97m:20052

[Ch1] Z. Chen, A classification of irreducible prehomogeneous vector spaces over an algebraically closed field of characteristic 2, I, Acta Math. Sinica, 2:168-177, 1986. MR 88f:20061

[Ch2] - A classification of irreducible prehomogeneous vector spaces over an algebraically closed field of characteristic p, II, Ann. Math. Ser. A, 9:10-22, 1988. MR 90g:20063

[CC] A. M. Cohen and B. N. Cooperstein, The 2-spaces of the standard E6 $(q)$-module, Geom. Ded., 25:467-480, 1988. MR 89g:51013

[GW] R. Gow and W. Willems, Methods to decide if simple self-dual modules over fields of characteristic 2 are of quadratic type, J. Algebra, 175:1067-1081, 1995. MR 96h:20017

[GS] R. M. Guralnick and G. M. Seitz, Irreducible subgroups of orthogonal and symplectic groups with finitely many orbits on singular $k$-spaces, in preparation, 1997.

[GLMS] R. M. Guralnick, M. W. Liebeck, H. D. Macpherson, and G. M. Seitz, Modules for algebraic groups with finitely many orbits, J. Algebra, 196:211-250, 1997. MR 98j:20058

[Hab] W. Haboush, Reductive groups are geometrically reductive, Ann. of Math., 102:67-84, 1975. MR 52:3179

[H1] J. E. Humphreys, Linear Algebraic Groups, Springer-Verlag, New York, third edition, 1987. MR 53:633

[H2] Introduction to Lie Algebras and Representation Theory, Springer-Verlag, New York, sixth edition, 1994. MR 81b:17007 
[Kac] V. G. Kac, Some remarks on nilpotent orbits, J. Algebra, 6:190-213, 1980. MR 81i:17005

[L] M. W. Liebeck, The affine permutation groups of rank three, Proc. London Math. Soc., 54:477-516, 1987. MR 88m:20004

[LSS] M. W. Liebeck, J. Saxl, and G. M. Seitz, Factorizations of simple algebraic groups, Trans. Amer. Math. Soc., 348:799-822, 1996. MR 96g:20064

[LS] M. W. Liebeck and G. M. Seitz, Reductive subgroups of exceptional algebraic groups, Mem. Amer. Math. Soc., No. 580, 1996. MR 96i:20059

[Lu] D. Luna, Sur les orbites fermées des groupes algébrique réductifs, Invent. Math., 16:1-5, 1972. MR 45:3421

[Ros] M. Rosenlicht, Some basic theorems on algebraic groups, Amer. J. Math., 78:401-443, 1956. MR 18:514a

[SK] M. Sato and T. Kimura, A classification of irreducible prehomogeneous vector spaces and their relative invariants, Nagoya Math. J., 65:1-155, 1977. MR 55:3341

[Se] G. M. Seitz, The maximal subgroups of classical algebraic groups, Mem. Amer. Math. Soc., No. 365, 1987. MR 88g:20092

[S1] R. Steinberg, Endomorphisms of linear algebraic groups, Mem. Amer. Math. Soc., No. 80, 1968. MR 37:6288

[S2] _ Conjugacy Classes in Algebraic Groups, volume 366 of Lecture Notes in Math., Springer, Berlin, 1974. MR 50:4766

Department of Mathematics, University of Oregon, Eugene, Oregon 97403-1222

E-mail address: brundan@darkwing.uoregon.edu 